\title{
Neutron pairing with medium polarization beyond the Landau approximation
}

\author{
M. Urban* \\ Institut de Physique Nucléaire, CNRS-IN2P3, Univ. Paris-Sud, \\ Université Paris-Saclay, 91406 Orsay cedex, France \\ S. Ramanan ${ }^{\dagger}$ \\ Department of Physics, Indian Institute of Technology Madras, Chennai - 600036, India
}

\begin{abstract}
We revisit the long-standing problem of the superfluid transition temperature $T_{c}$ in dilute neutron matter. It is well known that $T_{c}$ is strongly affected by medium polarization effects (screening) which modify the pairing interaction in the medium. We study these effects within the random-phase approximation (RPA). It turns out that the widely used Landau approximation is sufficient only at densities below about $0.002 \mathrm{fm}^{-3}$. At higher densities, the full RPA leads to stronger screening than the Landau approximation.
\end{abstract}

\section{INTRODUCTION}

Superfluidity in neutron stars is a long-standing problem that finds its inception in the attractive interaction between two nucleons that allows for the formation of Cooper pairs 1 3. Neutron stars, which are produced by a core-collapse supernova, are extremely dense objects, made of highly degenerate asymmetric nuclear matter. While the existence of a superfluid phase was theoretically predicted in the early 1960s [4, 5] and a value of $\sim 1 \mathrm{MeV}$ was correctly assigned to the pairing gap, a complete and systematic theoretical description is yet an open problem. The existence of this phase is significant from the point of view of cooling 6, 7] and is necessary to explain the observed glitches, which are sudden increases in the rotational frequency of the star, followed by long relaxation times to the pre-glitch values [8-10]. The attractive interaction is provided by the nucleon-nucleon interaction and therefore, by analyzing the two-body scattering phase shifts, one can conclude that neutrons can pair in the singlet, ${ }^{1} S_{0}$, channel at low densities, as they prevail in the neutron-star crust [1], and in the triplet, ${ }^{3} P_{2}-{ }^{3} F_{2}$, channels [12 15] at higher densities as they are expected in the neutron-star core. Protons pair up in the singlet channel because their density never gets very high. However, proton pairing is complicated by the interaction with the medium. In fact, medium effects are very important for neutron pairing as well.

Even when modelled as pure neutron matter, a correct description of the superfluid state is ridden with uncertainties. At low-densities, in the singlet channel, the BCS approximation gives almost model independent values for the transition temperature and gap, where by the BCS approximation, we refer to solving the BCS gap equation using free-space two-body interaction with a free single particle spectrum. Hence, at the BCS level, phase-shift equivalence of the two-body interaction suffices to yield model independent results [16]. However, description of

* urban@ipno.in2p3.fr

$\dagger$ suna@physics.iitm.ac.in

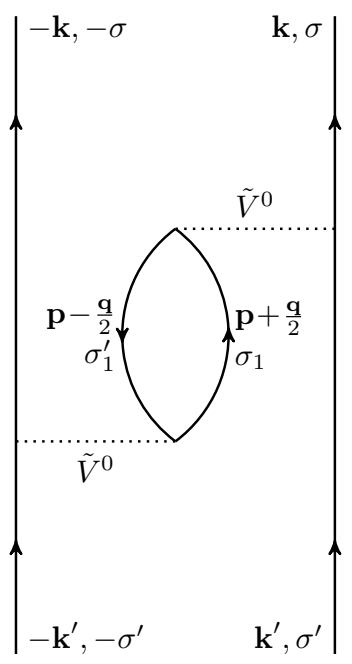

(a)

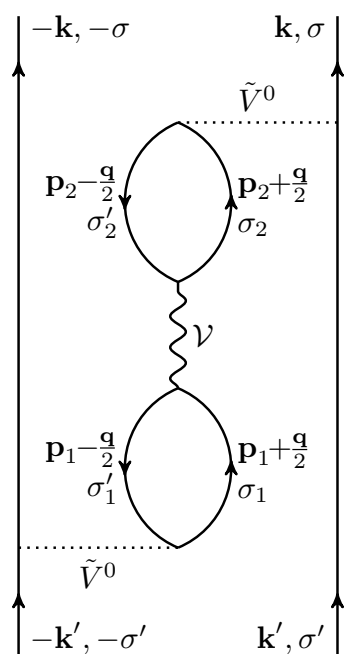

(b)
FIG. 1. Feynman diagrams representing the induced interaction. The wiggly line in diagram (b) is meant to include the RPA bubble summation (see Fig. 2 ).

pairing in the triplet channel, which typically occurs at higher densities becomes challenging even at the BCS level, as the input free-space two-body interactions are no-longer phase shift equivalent and hence the gaps turn out to be model dependent $12-15]$. As already noted, including the interaction of the neutrons with the medium is very important and can lead to crucial medium modifications such as screening of the free-space two-body interaction. However, the gap and the transition temperature are extremely sensitive to the approximation used to describe medium effects.

In our previous work [17, the free-space interaction was modified by including the effects of diagrams (a) and (b) in Fig. 2. There, we used the free-space renormalized two-body interaction, $V_{\text {low } k}$, for the bare pairing interaction (that is, particle-particle vertex, without medium correction) and for the $3 \mathrm{p} 1 \mathrm{~h}$ (3-particle-1-hole) and 1p3h interactions, while the RPA (random-phase approxima- 


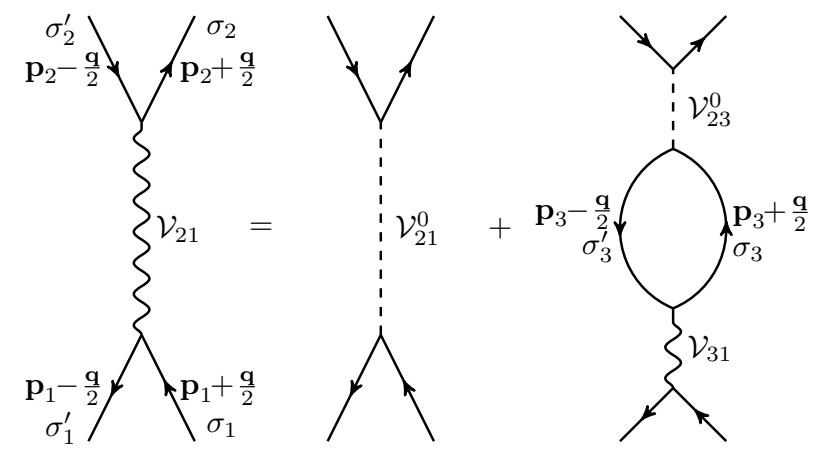

FIG. 2. Diagrammatic representation of the RPA, Eq. (12).

tion) series (see Fig. 2) used the particle-hole (ph) interaction within the Landau approximation. The Fermiliquid parameters were obtained using phenomenological (Skyrme, Gogny) energy-density functionals. The inclusion of diagram (b) is important at higher densities since it reduces the effect of diagram (a). In fact, it even results in the gap being anti-screened for $k_{F} \gtrsim 0.7 \mathrm{fm}^{-1}$ $\left(k_{F}\right.$ denotes the Fermi momentum which is related to the density by $\rho=k_{F}^{3} / 3 \pi^{2}$ for pure neutron matter). While the result that the effect of screening is reduced as compared to diagram (a) is consistent with Quantum Monte Carlo (QMC) calculations [18, 20, it is important to check the dependence of the contribution from diagram (b) on the approximation used. To our knowledge, all present calculations of medium-polarization effects that include the RPA resummation, e.g., 21 24], rely on the Landau approximation. As already noted in [17, the Landau approximation is valid for momentum transfers $q \ll k_{F}$, which is clearly not the case in diagram (b) where $0 \leq q \lesssim 2 k_{F}$.

In this paper, we once again revisit the issue of medium polarization in pure neutron matter. We continue to use the $V_{\text {low } k}$ interaction for the bare pairing interaction and for the $3 \mathrm{p} 1 \mathrm{~h}$ and $1 \mathrm{p} 3 \mathrm{~h}$ vertices. But now, we evaluate the RPA series in diagram (b) exactly, i.e., beyond the Landau approximation. This is possible since we use ph interactions of the Skyrme type. We repeat our calculations with different Skyrme parameterizations (SLy4 and SLy5 from the Saclay-Lyon family 25] and BSk19, BSk20, and BSk21 from the recent Brussels-Montreal family [26]) so that we can assess the dependence of the result on the choice of the parameterization.

This paper is organized as follows. In Sec.II. we revisit the set up of diagrams (a) and (b), followed by the calculation of RPA diagrams using Skyrme interactions in Sec. III We discuss in detail the choice of the cutoff for the $V_{\text {low } k}$ interaction and also the parameters used for the Skyrme interaction in Sec. IV] In Sec. V], we present the main results of the paper. We study the dependence of the screened interaction on the Skyrme parameterization as well as compare our results to those obtained in [17. We also revisit the effect of preformed pairs on the den- sity dependence of the transition temperature. Finally, in Sec. VI, we present our concluding remarks. Some details of the calculations have been moved to appendices.

\section{PAIRING WITH MEDIUM POLARIZATION}

In general, to obtain the ${ }^{1} S_{0}$ pairing gap $\Delta$ or the critical temperature $T_{c}$, one considers the gap equation

$$
\Delta_{k}=-\frac{2}{\pi} \int_{0}^{\infty} d k^{\prime} k^{\prime 2} V\left(k, k^{\prime}\right) \frac{\Delta_{k^{\prime}} \tanh \left(\frac{E_{k^{\prime}}}{2 T}\right)}{2 E_{k^{\prime}}}
$$

where $V\left(k, k^{\prime}\right)=\left\langle k\left|V_{{ } S_{0}}\right| k^{\prime}\right\rangle$ denotes the matrix element of the $n n$ interaction in the ${ }^{1} S_{0}$ partial wave for in- and outgoing momenta $k^{\prime}$ and $k, E_{k}=\sqrt{\left(\epsilon_{k}-\mu\right)^{2}+\Delta_{k}^{2}}$ is the quasiparticle energy with $\epsilon_{k}=k^{2} / 2 m^{*}, m^{*}$ is the neutron effective mass, $\mu$ is the effective chemical potential including the mean-field energy shift, and $T$ is the temperature. Including medium polarization effects in a way analogous to Debye screening of the Coulomb interaction [27], the interaction $V\left(k, k^{\prime}\right)$ can be written as

$$
V\left(k, k^{\prime}\right)=V^{0}\left(k, k^{\prime}\right)+V^{(a)}\left(k, k^{\prime}\right)+V^{(b)}\left(k, k^{\prime}\right),
$$

where $V^{0}$ is the contribution of the bare $n n$ interaction, $V^{(a)}$ is the contribution of a single ph bubble exchange, and $V^{(b)}$ represents the RPA resummation of the series of two and more bubbles, see Fig. 1] To evaluate $V^{(a)}$, we proceed as in [17] and construct the bare interaction from $V_{\text {low } k}$ [28, given in partial waves, as

$$
\begin{aligned}
& \left\langle\mathbf{k}_{1} \sigma_{1}, \mathbf{k}_{2} \sigma_{2}\left|V^{0}\right| \mathbf{k}_{1}^{\prime} \sigma_{1}^{\prime}, \mathbf{k}_{2}^{\prime} \sigma_{2}^{\prime}\right\rangle= \\
& \sum_{s, m_{s}, m_{s}^{\prime}} \sum_{l, l^{\prime}, m_{l}} \sum_{j} C_{\frac{1}{2} \sigma_{1} \frac{1}{2} \sigma_{2}}^{s m_{s}} C_{\frac{1}{2} \sigma_{1}^{\prime} \frac{1}{2} \sigma_{2}^{\prime}}^{s m_{l}^{\prime}} C_{l m_{l} s m_{s}}^{j m_{j}} C_{l^{\prime} m_{l}^{\prime} s m_{s}^{\prime}}^{j m_{j}} \\
& \quad \times(4 \pi)^{2} i^{l^{\prime}-l} Y_{l m_{l}}^{*}\left(\Omega_{\mathbf{Q}}\right) Y_{l^{\prime} m_{l}^{\prime}}\left(\Omega_{\mathbf{Q}^{\prime}}\right)\left\langle Q\left|V_{s l l^{\prime} j}^{0}\right| Q^{\prime}\right\rangle
\end{aligned}
$$

with $m_{l}^{\prime}=m_{l}+m_{s}-m_{s}^{\prime}, m_{j}=m_{l}+m_{s}, \mathbf{Q}=\left(\mathbf{k}_{1}-\mathbf{k}_{2}\right) / 2$, and $\mathbf{Q}^{\prime}=\left(\mathbf{k}_{1}^{\prime}-\mathbf{k}_{2}^{\prime}\right) / 2$. For the Clebsch-Gordan coefficients, we follow the notation of the book by Varshalovich [29]. The antisymmetrized interaction is defined by

$$
\left\langle 1,2\left|\tilde{V}^{0}\right| 1^{\prime}, 2^{\prime}\right\rangle=\left\langle 1,2\left|V^{0}\right| 1^{\prime}, 2^{\prime}\right\rangle-\left\langle 1,2\left|V^{0}\right| 2^{\prime}, 1^{\prime}\right\rangle
$$

where the short-hand notation 1 stands for $\mathbf{k}_{1} \sigma_{1}$ etc.

The expression corresponding to diagram (a) reads

$$
\begin{aligned}
& V^{(a)}\left(k, k^{\prime}\right)=\frac{-1}{4 \pi} \sum_{\sigma \sigma^{\prime}} C_{\frac{1}{2} \sigma \frac{1}{2}-\sigma}^{00} C_{\frac{1}{2} \sigma^{\prime} \frac{1}{2}-\sigma^{\prime}}^{00} \int \frac{d \Omega_{k}}{4 \pi} \int \frac{d \Omega_{k^{\prime}}}{4 \pi} \\
& \times \sum_{\mathbf{p} \sigma_{1}}\left\langle-\mathbf{k}-\sigma, \mathbf{p}+\frac{\mathbf{q}}{2} \sigma_{1}\left|\tilde{V}^{0}\right|-\mathbf{k}^{\prime}-\sigma^{\prime}, \mathbf{p}-\frac{\mathbf{q}}{2} \sigma_{1}^{\prime}\right\rangle \\
& \times \frac{n_{\mathbf{p}-\frac{\mathbf{q}}{2}}-n_{\mathbf{p}+\frac{\mathbf{q}}{2}}}{\epsilon_{\mathbf{p}+\frac{\mathbf{q}}{2}}-\epsilon_{\mathbf{p}-\frac{\mathbf{q}}{2}}}\left\langle\mathbf{p}-\frac{\mathbf{q}}{2} \sigma_{1}^{\prime}, \mathbf{k} \sigma\left|\tilde{V}^{0}\right| \mathbf{p}+\frac{\mathbf{q}}{2} \sigma_{1}, \mathbf{k}^{\prime} \sigma^{\prime}\right\rangle .
\end{aligned}
$$

where $\mathbf{q}=\mathbf{k}-\mathbf{k}^{\prime}$ and $\sum_{\mathbf{p}}$ stands for $\int d^{3} p /(2 \pi)^{3}$. The occupation numbers $n_{\mathbf{p}}$ can be safely approximated by 
step functions, $n_{\mathbf{p}}=\theta\left(k_{F}-p\right)$, because the temperature $T$ and the pairing gap $\Delta$ are much smaller than the Fermi energy $\epsilon_{F}$.

Similarly, the expression for diagram (b) can be written as

$$
\begin{aligned}
& V^{(b)}\left(k, k^{\prime}\right)=\frac{1}{4 \pi} \sum_{\sigma \sigma^{\prime}} C_{\frac{1}{2} \sigma \frac{1}{2}-\sigma}^{00} C_{\frac{1}{2} \sigma^{\prime} \frac{1}{2}-\sigma^{\prime}}^{00} \int \frac{d \Omega_{q}}{4 \pi} \int \frac{d \Omega_{q^{\prime}}}{4 \pi} \\
& \times \sum_{\mathbf{p}_{1} \sigma_{1}} \sum_{\mathbf{p}_{2} \sigma_{2}}\left\langle-\mathbf{k}-\sigma, \mathbf{p}_{1}+\frac{\mathbf{q}}{2} \sigma_{1}\left|\tilde{V}^{0}\right|-\mathbf{k}^{\prime}-\sigma^{\prime}, \mathbf{p}_{1}-\frac{\mathbf{q}}{2} \sigma_{1}^{\prime}\right\rangle \\
& \quad \times \frac{n_{\mathbf{p}_{1}-\frac{\mathbf{q}}{2}}-n_{\mathbf{p}_{1}+\frac{\mathbf{q}}{2}}}{\epsilon_{\mathbf{p}_{1}+\frac{\mathbf{q}}{2}}-\epsilon_{\mathbf{p}_{1}-\frac{\mathbf{q}}{2}}} \mathcal{V}_{\mathbf{p}_{2}-\frac{\mathbf{q}}{2} \sigma_{2}^{\prime}, \mathbf{p}_{2}+\frac{\mathbf{q}}{2} \sigma_{2} ; \mathbf{p}_{1}+\frac{\mathbf{q}}{2} \sigma_{1}, \mathbf{p}_{1}-\frac{\mathbf{q}}{2} \sigma_{1}^{\prime}} \\
& \times \frac{n_{\mathbf{p}_{2}-\frac{\mathbf{q}}{2}}-n_{\mathbf{p}_{2}+\frac{\mathbf{q}}{2}}}{\epsilon_{\mathbf{p}_{2}+\frac{\mathbf{q}}{2}}-\epsilon_{\mathbf{p}_{2}-\frac{\mathbf{q}}{2}}}\left\langle\mathbf{p}_{2}-\frac{\mathbf{q}}{2} \sigma_{2}^{\prime}, \mathbf{k} \sigma\left|\tilde{V}^{0}\right| \mathbf{p}_{2}+\frac{\mathbf{q}}{2} \sigma_{2}, \mathbf{k}^{\prime} \sigma^{\prime}\right\rangle .
\end{aligned}
$$

Here, $\mathcal{V}$ denotes the ph interaction including the resummation of bubble diagrams. It is different from the particle-particle (pp) interaction $V^{0}$ and we therefore use a different notation. Like the effective mass $m^{*}$, the ph interaction will not be derived from the free-space interaction $V^{0}$, but from a phenomenological Skyrme energy functional. Previous studies of screening used the simplest approximation to $\mathcal{V}$, namely the lowest-order Landau approximation

$$
\begin{aligned}
& \mathcal{V}_{\mathbf{p}_{2}-\frac{\mathrm{q}}{2} \sigma_{2}^{\prime}, \mathbf{p}_{2}+\frac{\mathbf{q}}{2} \sigma_{2} ; \mathbf{p}_{1}+\frac{\mathrm{q}}{2} \sigma_{1}, \mathbf{p}_{1}-\frac{\mathrm{q}}{2} \sigma_{1}^{\prime}} \approx \frac{f_{0} \delta_{\sigma_{1}^{\prime} \sigma_{1}} \delta_{\sigma_{2} \sigma_{2}^{\prime}}}{1-f_{0} \Pi_{0}(q)} \\
&+\frac{g_{0} \boldsymbol{\sigma}_{\sigma_{1}^{\prime} \sigma_{1}} \cdot \boldsymbol{\sigma}_{\sigma_{2} \sigma_{2}^{\prime}}}{1-g_{0} \Pi_{0}(q)},
\end{aligned}
$$

where $f_{0}$ and $g_{0}$ are the $L=0$ Landau parameters in the density and spin channel, respectively, $\sigma$ are the Pauli matrices, and $\Pi_{0}(q)$ is the Lindhard function in the static limit, see Appendix C. Inserting Eq. (7) into Eq. (6) and renaming $\mathbf{k}, \mathbf{k}^{\prime}, \mathbf{q}, \mathbf{p}_{i} \rightarrow \mathbf{q}, \mathbf{q}^{\prime}, \mathbf{k}, \mathbf{p}_{i}-\mathbf{k} / 2$, one recovers the expression given in our previous work [17. The aim of the present study is to go beyond this approximation and to include the RPA calculated with the full Skyrme ph interaction.

\section{RPA WITH SKYRME INTERACTION}

The RPA with Skyrme interactions has been extensively studied, see the review [30]. However, in most calculations, one is interested in the response function and not in the vertex $\mathcal{V}$ which we need here. The RPA vertex function for Skyrme-like interactions was, e.g., considered in 31 .

The residual ph interaction is derived from the Skyrme energy functional $E$ as 31

$$
\begin{aligned}
\mathcal{V}_{\mathbf{p}_{2}-\frac{\mathbf{q}}{2} \sigma_{2}^{\prime}, \mathbf{p}_{2}+\frac{\mathbf{q}}{2} \sigma_{2} ; \mathbf{p}_{1}+\frac{\mathbf{q}}{2} \sigma_{1}, \mathbf{p}_{1}-\frac{\mathbf{q}}{2} \sigma_{1}^{\prime}} & =\frac{\delta^{2} E}{\delta \rho_{\mathbf{p}_{2}-\frac{\mathbf{q}}{2} \sigma_{2}^{\prime}, \mathbf{p}_{2}+\frac{\mathbf{q}}{2} \sigma_{2}} \delta \rho_{\mathbf{p}_{1}+\frac{\mathbf{q}}{2} \sigma_{1}, \mathbf{p}_{1}-\frac{\mathbf{q}}{2} \sigma_{1}^{\prime}}},
\end{aligned}
$$

where $\rho_{\mathbf{p} \sigma, \mathbf{p}^{\prime}, \sigma^{\prime}}$ denotes the density matrix with the momentum and spin indices as defined in Fig. 2, Let us introduce the short-hand notation

$$
\begin{gathered}
\mathcal{V}_{21}^{0}=\mathcal{V}_{\mathbf{p}_{2}-\frac{\mathbf{q}}{2} \sigma_{2}^{\prime}, \mathbf{p}_{2}+\frac{\mathbf{q}}{2} \sigma_{2} ; \mathbf{p}_{1}+\frac{\mathbf{q}}{2} \sigma_{1}, \mathbf{p}_{1}-\frac{\mathbf{q}}{2} \sigma_{1}^{\prime},}, \\
\boldsymbol{\sigma}_{1}=\boldsymbol{\sigma}_{\sigma_{1}^{\prime} \sigma_{1}},
\end{gathered}
$$

etc., where 1 stands for the quantum numbers $\mathbf{p}_{1}, \sigma_{1}, \sigma_{1}^{\prime}$ and similarly for 2 . If no spin operator $\boldsymbol{\sigma}_{1}$ is written, the corresponding term is assumed to be proportional to $\delta_{\sigma_{1}^{\prime} \sigma_{1}}$. Using this notation, the interaction derived from a standard Skyrme functional has the form

$$
\begin{aligned}
\mathcal{V}_{21}^{0}=v_{1}^{0}(q)+v_{2}^{0}\left(\mathbf{p}_{1}-\mathbf{p}_{2}\right)^{2} & \\
+\left[v_{4}^{0}(q)\right. & \left.+v_{5}^{0}\left(\mathbf{p}_{1}-\mathbf{p}_{2}\right)^{2}\right] \boldsymbol{\sigma}_{1} \cdot \boldsymbol{\sigma}_{2} \\
& +v_{8}^{0} i \mathbf{q} \cdot\left(\mathbf{p}_{1}-\mathbf{p}_{2}\right) \times\left(\boldsymbol{\sigma}_{1}+\boldsymbol{\sigma}_{2}\right) .
\end{aligned}
$$

The $v_{i}^{0}$ can be density dependent and their expressions in terms of the parameters of the Skyrme force are given in Appendix A Notice that, as a consequence of its derivation via Eq. (8), $\mathcal{V}_{21}^{0}$ contains already both the direct and the exchange term.

The RPA vertex $\mathcal{V}$ is obtained from the ph interaction $\mathcal{V}^{0}$ by solving the Bethe-Salpeter like equation (see Fig. 2p

$$
\mathcal{V}_{21}=\mathcal{V}_{21}^{0}-\sum_{3} \mathcal{V}_{23}^{0} G_{\mathrm{ph}}^{0}\left(\mathbf{p}_{3}, \mathbf{q}\right) \mathcal{V}_{31}
$$

where the minus sign comes from the closed fermion loop, $\sum_{3}$ is a short-hand notation for $\sum_{\sigma_{3} \sigma_{3}^{\prime}} \int d^{3} p_{3} /(2 \pi)^{3}$, and

$$
G_{\mathrm{ph}}^{0}(\mathbf{p}, \mathbf{q})=\frac{n_{\mathbf{p}-\frac{\mathrm{q}}{2}}-n_{\mathbf{p}+\frac{\mathrm{q}}{2}}}{\epsilon_{\mathbf{p}+\frac{\mathrm{q}}{2}}-\epsilon_{\mathbf{p}-\frac{\mathrm{q}}{2}}}
$$

is the ph propagator in the static limit, i.e., for $\omega \rightarrow 0$, where $\omega$ is the total energy of the ph pair. While Eq. 122 is in general quite difficult to solve, it is very simple in the case of a Skyrme interaction.

The RPA vertex has a more general structure than the Skyrme ph interaction in Eq. (11). Nevertheless, since all terms in the Skyrme force are at most quadratic in $\mathbf{p}_{1}$ and $\mathbf{p}_{2}$, only a finite number of terms are generated [31. The number of independent terms is further reduced because the vertex is Hermitian, i.e., $\mathcal{V}_{21}=\mathcal{V}_{12}^{*}$, and the dependence on the angles of $\mathbf{p}_{1}$ and $\mathbf{p}_{2}$ is at most $L=1$. In the case of a standard Skyrme interaction, it turns out that the following ansatz for $\mathcal{V}$ is sufficient:

$$
\begin{gathered}
\mathcal{V}_{21}=v_{1}+v_{2}\left(p_{1}^{2}+p_{2}^{2}\right)+v_{3} \mathbf{p}_{1} \cdot \mathbf{p}_{2}+v_{4} \boldsymbol{\sigma}_{1} \cdot \boldsymbol{\sigma}_{2} \\
+v_{5} \boldsymbol{\sigma}_{1} \cdot \boldsymbol{\sigma}_{2}\left(p_{1}^{2}+p_{2}^{2}\right)+v_{6} \boldsymbol{\sigma}_{1} \cdot \boldsymbol{\sigma}_{2} \mathbf{p}_{1} \cdot \mathbf{p}_{2}+v_{7} \boldsymbol{\sigma}_{1} \cdot \mathbf{q} \boldsymbol{\sigma}_{2} \cdot \mathbf{q} \\
+v_{8} i \mathbf{q} \cdot\left(\mathbf{p}_{1} \times \boldsymbol{\sigma}_{1}-\mathbf{p}_{2} \times \boldsymbol{\sigma}_{2}\right)+v_{9} i \mathbf{q} \cdot\left(\mathbf{p}_{1} \times \boldsymbol{\sigma}_{2}-\mathbf{p}_{2} \times \boldsymbol{\sigma}_{1}\right) \\
+v_{10} p_{1}^{2} p_{2}^{2}+v_{11} \mathbf{p}_{1} \cdot \mathbf{q} \mathbf{p}_{2} \cdot \mathbf{q}+v_{12} \boldsymbol{\sigma}_{1} \cdot \boldsymbol{\sigma}_{2} p_{1}^{2} p_{2}^{2} \\
+v_{13} \boldsymbol{\sigma}_{1} \cdot \boldsymbol{\sigma}_{2} \mathbf{p}_{1} \cdot \mathbf{q} \mathbf{p}_{2} \cdot \mathbf{q}+v_{14} \boldsymbol{\sigma}_{1} \cdot \mathbf{q} \boldsymbol{\sigma}_{2} \cdot \mathbf{q}\left(p_{1}^{2}+p_{2}^{2}\right) \\
+v_{15} \boldsymbol{\sigma}_{1} \cdot \mathbf{q} \boldsymbol{\sigma}_{2} \cdot \mathbf{q} p_{1}^{2} p_{2}^{2}+v_{16} i \mathbf{q} \cdot\left(\mathbf{p}_{1} \times \boldsymbol{\sigma}_{1} p_{2}^{2}-\mathbf{p}_{2} \times \boldsymbol{\sigma}_{2} p_{1}^{2}\right) \\
+v_{17} i \mathbf{q} \cdot\left(\mathbf{p}_{1} \times \boldsymbol{\sigma}_{2} p_{2}^{2}-\mathbf{p}_{2} \times \boldsymbol{\sigma}_{1} p_{1}^{2}\right) \\
+v_{18} \mathbf{q} \cdot \mathbf{p}_{1} \times \boldsymbol{\sigma}_{1} \mathbf{q} \cdot \mathbf{p}_{2} \times \boldsymbol{\sigma}_{2} \cdot \quad \text { (14) }
\end{gathered}
$$


The coefficients $v_{i}$ are functions of $q$ and $\rho$, but we drop the arguments for brevity. In order to determine the functions $v_{i}$, we insert Eqs. (11) and (14) into Eq. (12). The integrals over $\mathbf{p}_{3}$ can be expressed in terms of the generalized Lindhard functions

$$
\begin{gathered}
\Pi_{n}(q)=-2 \int \frac{d^{3} p}{(2 \pi)^{3}} p^{n} G_{\mathrm{ph}}, \\
\Pi_{2 L}(q)=-2 \int \frac{d^{3} p}{(2 \pi)^{3}} p^{2} \cos ^{2} \theta G_{\mathrm{ph}}, \\
\Pi_{2 T}(q)=-\int \frac{d^{3} p}{(2 \pi)^{3}} p^{2} \sin ^{2} \theta G_{\mathrm{ph}}=\frac{\Pi_{2}-\Pi_{2 L}}{2},
\end{gathered}
$$

$\theta$ being the angle between $\mathbf{p}$ and $\mathbf{q}$. The explicit expressions for these functions are given in Appendix C. Collecting the coefficients of the different operators that appear in Eq. (14), one obtains a linear system of equations for the $v_{i}$ of the form

$$
v_{i}=v_{i}^{0}+\sum_{j} A_{i, j} v_{j}
$$

where the matrix elements $A_{i, j}$ are products of the different $v_{i}^{0}$ and $\Pi_{i}$. Solving this system of equations, one obtains analytical expressions for the $v_{i}$, which are listed in Appendix D.

To get a qualitative idea about the difference between the full RPA and the Landau approximation, let us consider the static $(\omega \rightarrow 0)$ density response (ph $\operatorname{spin} S=0$ )

$$
\begin{aligned}
\Pi_{\mathrm{RPA}}^{(0)}=- & \sum_{1} G_{\mathrm{ph}}^{0}\left(\mathbf{p}_{1}, \mathbf{q}\right) \\
& +\sum_{1,2} G_{\mathrm{ph}}^{0}\left(\mathbf{p}_{1}, \mathbf{q}\right) V_{12} G_{\mathrm{ph}}^{0}\left(\mathbf{p}_{2}, \mathbf{q}\right) \\
& =\Pi_{0}+v_{1} \Pi_{0}^{2}+2 v_{2} \Pi_{0} \Pi_{2}+v_{10} \Pi_{2}^{2} .
\end{aligned}
$$

Similarly, one obtains the spin response $(S=1)$ by including Pauli matrices into the sums over 1 and 2 . The result for the transverse spin response $(M= \pm 1$, where $M$ denotes the projection of the ph spin on the direction of $\mathbf{q})$ takes the form

$$
\Pi_{\mathrm{RPA}}^{(1, \pm 1)}=\Pi_{0}+v_{4} \Pi_{0}^{2}+2 v_{5} \Pi_{0} \Pi_{2}+v_{12} \Pi_{2}^{2},
$$

whereas additional terms appear in the case of the longitudinal spin response $(S=1, M=0)$ :

$$
\Pi_{\mathrm{RPA}}^{(1,0)}=\Pi_{\mathrm{RPA}}^{(1, \pm 1)}+q^{2}\left(v_{7} \Pi_{0}^{2}+2 v_{14} \Pi_{0} \Pi_{2}+v_{15} \Pi_{2}^{2}\right) .
$$

In the absence of tensor terms, which we have not considered here because they do not appear in standard Skyrme interactions, these expressions agree with Eqs. (53), (55), and (56) of Ref. 30]. For comparison, in Landau approximation, one has $v_{1}=f_{0} /\left(1-f_{0} \Pi_{0}\right), v_{4}=g_{0} /\left(1-g_{0} \Pi_{0}\right)$, and all other $v_{i}=0$, and therefore

$$
\Pi_{\text {Landau }}^{(S=0)}=\frac{\Pi_{0}}{1-f_{0} \Pi_{0}}, \quad \Pi_{\text {Landau }}^{(S=1)}=\frac{\Pi_{0}}{1-g_{0} \Pi_{0}} .
$$

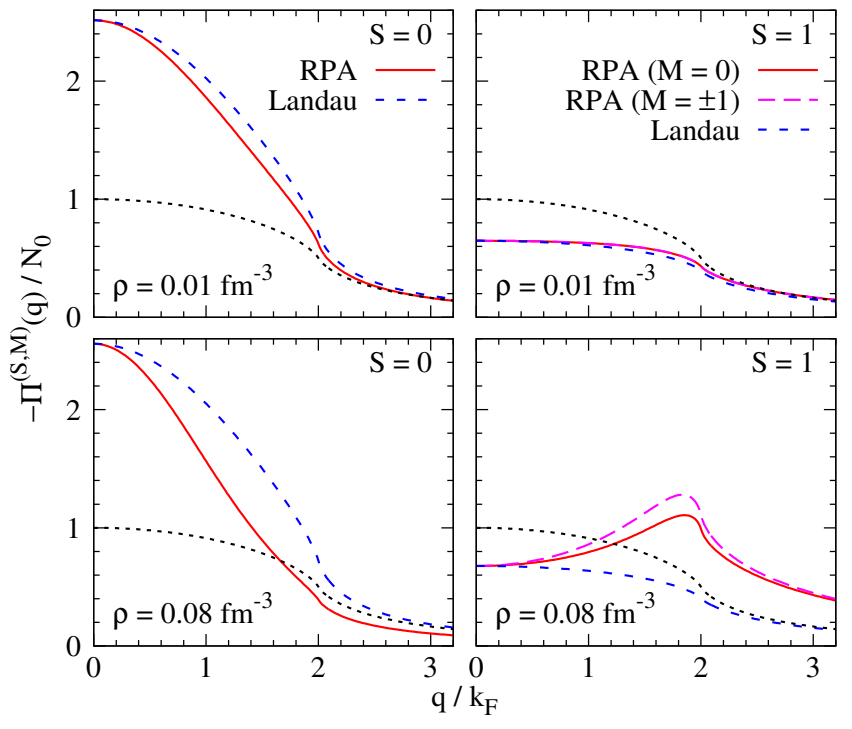

FIG. 3. Density response ( $S=0$, left column) and longitudinal $(M=0)$ and transverse $(M= \pm 1)$ spin responses $(S=1$, right column) in units of the density of states $N_{0}=m^{*} k_{F} / \pi^{2}$ as functions of $q / k_{F}$, for two different densities: $\rho=0.01 \mathrm{fm}^{-3}$ $\left(k_{F}=0.67 \mathrm{fm}^{-1}\right.$, upper row $)$ and $0.08 \mathrm{fm}^{-3}\left(k_{F}=1.33 \mathrm{fm}^{-1}\right.$, lower row), obtained with the SLy5 interaction. Results of the full RPA (red solid line and long purple dashes) are compared with those of the Landau approximation (short blue dashes) and with the Lindhard function $-\Pi_{0}$ (black dotted line).

The full RPA responses and the responses in Landau approximation are shown in Fig. 3 for two different densities. At low density ( $\rho=0.01 \mathrm{fm}^{-3}$, upper panels), the Landau approximation reproduces quite well the full RPA result. This is because the integration region of the internal loop momenta $p$ as well as the relevant range of external momenta $q$ scale with $k_{F}$, so that the momentum dependent terms of the full Skyrme interaction $\mathcal{V}^{0}$ are small at low density. Nevertheless, one can see that with increasing momentum the RPA responses are closer to the Hartree-Fock response (Lindhard function $\Pi_{0}$ ) than the Landau approximation. This reflects the fact that, roughly speaking, $\mathcal{V}^{0}$ becomes smaller with increasing momentum, as one would expect for a finite-range interaction which is simulated by the momentum dependence of the Skyrme force. At higher density $\left(\rho=0.08 \mathrm{fm}^{-3}\right.$, lower panels of Fig. 3), the momentum dependence of the interaction is so strong that it even changes sign. As a consequence, the density response (left panel) falls below $\Pi_{0}$ at large $q / k_{F}$, while the spin response (right panel) becomes enhanced compared to $\Pi_{0}$. It is not clear whether this is realistic or just an artefact of the limitation of the Skyrme force to terms up to second order in the momenta.

It would be interesting to compare these results with those obtained with a true finite-range interaction such as the Gogny force. However, in that case the solution of the RPA is very difficult and this is beyond the scope of the present study. QMC calculations 32 seem to indicate 
that the static density response of neutron matter should approach the free one (i.e., $\Pi_{0}$ but computed without effective mass; note that in general $m^{*}$ is momentum dependent) at large $q$.

\section{CHOICE OF INTERACTIONS AND PARAMETERS}

Before presenting numerical results, let us specify the choice of the interaction $V^{0}$ in the pp channel, which is also used in the $3 \mathrm{p} 1 \mathrm{~h}$ and $1 \mathrm{p} 3 \mathrm{~h}$ vertices in diagrams (a) and (b), and of the parameterizations of the Skyrme interaction $\mathcal{V}^{0}$ used in the calculation of the effective mass $m^{*}$ and in the ph channel for the RPA.

As bare interaction $V^{0}$, we use the low-momentum interaction $V_{\text {low } k}$ derived from the $\mathrm{AV}_{18}$ interaction by a renormalization-group evolution [28. The partial waves [cf. Eq. [30] are summed up to $j_{\max }=3$ which for our purposes is sufficient to reach converged results [17].

The $V_{\text {low } k}$ interaction has an additional parameter, namely the momentum cutoff $\Lambda$. Although, by construction, $V_{\text {low } k}$ gives cutoff-independent results in the twobody sector (at sufficiently low energies), this is not the case if it is used in a many-body calculation, where the dependence on the cutoff indicates missing medium and higher-body corrections. Actually, the purpose of using $V_{\text {low } k}$ instead of $\mathrm{AV}_{18}$ is to make the interaction "more perturbative" and thus more suitable for approximations used in the many-body problem. A common choice is a cutoff $\Lambda=2 \mathrm{fm}^{-1}$ which we will also use here, especially at densities with $k_{F} \gtrsim 0.8 \mathrm{fm}^{-1}$.

However, as explained in detail in Ref. [17, to reproduce the correct low-density limit of the screening correction (Gor'kov-Melik-Barkhudarov (GMB) result 33]), it is important that the $3 \mathrm{p} 1 \mathrm{~h}$ and $1 \mathrm{p} 3 \mathrm{~h}$ vertices approach the $n n$ scattering length, while in diagrams (a) and (b) these vertices contain the interaction only to leading order (Born term). We remind the reader that in the limit of small cutoffs $\Lambda$ and small momenta $k$ and $k^{\prime}$, the interaction $V^{0}\left(k, k^{\prime}\right)$, the cutoff $\Lambda$, and the scattering length $a$ are roughly related by

$$
V^{0} \sim\left(\frac{m}{a}-\frac{2 m \Lambda}{\pi}\right)^{-1} .
$$

Hence, by lowering the cutoff as much as possible, we can achieve $V^{0} \sim a / m$. Obviously, the cutoff must remain larger than $k_{F}$ if one wants to describe Cooper pairing. In practice, when solving the gap equation with $V^{0}$ alone, one can decrease the cutoff to $\Lambda=2.5 k_{F}$ (with a regulator of the form $e^{\left.-\left(k^{2} / \Lambda^{2}\right)^{5}\right)}$ without affecting the critical temperature. As in Ref. [17, we will use this as an alternative choice, especially for low densities with $k_{F} \lesssim 0.8 \mathrm{fm}^{-1}$.

The reason why we do not use the density dependent cutoff at higher values of $k_{F}$ is the following. As $k_{F}$ increases, the variable cutoff grows and as a result will include the coupling between the low- and high-momentum physics, making the interaction less useful in perturbation theory. The BCS transition temperatures and gaps in the singlet channel are unaffected by the coupling between low- and high-momentum modes, as they depend on the correct reproduction of the two-body scattering data. But the perturbativeness of the interaction becomes important in the $3 \mathrm{p} 1 \mathrm{~h}$ and $1 \mathrm{p} 3 \mathrm{~h}$ vertices, and also in the Nozières-Schmitt-Rink (NSR) approach (see next section and Appendix E), where one has to compensate for the double counting of the single-particle energy shift (see [34), which in our case is done within the HartreeFock approximation. Therefore, it is crucial to soften the interaction. Hence we use a variable cutoff of $2.5 k_{F}$ until $k_{F} \approx 0.8 \mathrm{fm}^{-1}$ and a constant cutoff of $2 \mathrm{fm}^{-1}$ for higher densities.

Concerning the ph interaction, there are hundreds of different parameterizations of the Skyrme interaction on the market, which were fitted in different ways and for different purposes. Fortunately, the number of interactions suitable for neutron matter is much smaller. The first interactions that were fitted to reproduce not only finite nuclei but also infinite neutron matter were those of the Saclay-Lyon (SLy...) family. We will use the parameterizations SLy4 and SLy5 25]. More recent Skyrme interactions developed specifically for astrophysical applications are those of the Brussels-Montreal (BSk...) family. Out of this family, we will use the parameterizations BSk19, BSk20, and BSk21 [26]. To use several different parameterizations can be useful to get an idea how strongly the results depend on this choice.

The Fermi-liquid parameters corresponding to these interactions (see Appendix B) are shown in Fig. 4. In our preceding work [17, we computed the Fermi-liquid parameters from the SLy4 parameterization, but using $\eta_{J}=1$ in the calculation of $G_{0}$, although the SLy4 functional was fitted without the $\mathbb{J}^{2}$ terms, i.e., with $\eta_{J}=0$ (following the notations of [35]). This was somewhat inconsistent (although quite common in the literature). Here, by using the SLy5 parameterization, which was fitted with $\eta_{J}=1$, we avoid this inconsistency and we still find Fermi liquid parameters (blue short dashes) very close to those shown in our preceding work (Fig. 3 of Ref. [17]). On the contrary, the SLy4 results shown here (red solid lines) are now obtained with $\eta_{J}=0$ which explains why the $G_{0}$ Landau parameter is much more repulsive than with SLy5.

In Ref. [36, it was pointed out that the $G_{0}$ parameter of SLy5 is probably too small, which can lead to ferromagnetic instabilities at higher densities. It was therefore suggested that one should rather use SLy4 (with $\eta_{J}=0$ ) or more recent parameterizations such as BSk19-21 which were fitted (again with $\eta_{J}=0$ ) to give a reasonable $G_{0}$ at saturation density. But even the latter parameterizations lead to instabilities in the spin channel for neutron matter above saturation density $0.16 \mathrm{fm}^{-3}[37$. 

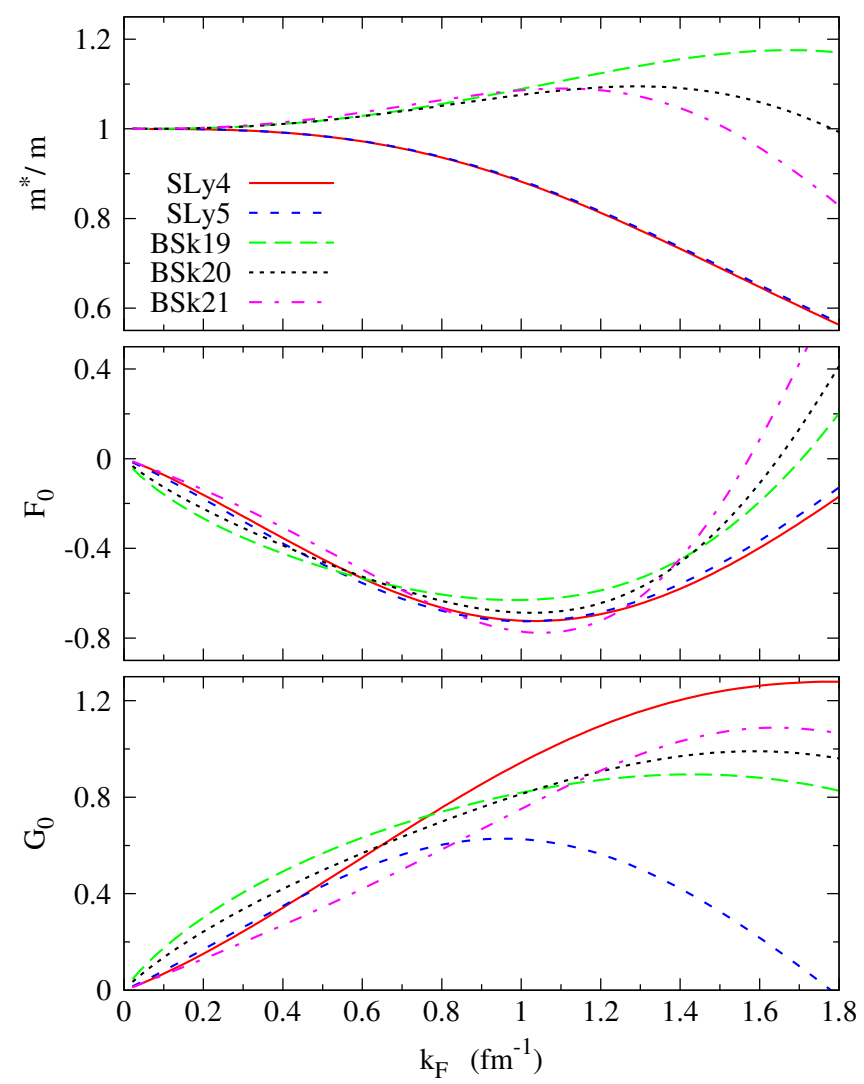

FIG. 4. Fermi-liquid parameters $m^{*}, F_{0}$, and $G_{0}$ as functions of the Fermi momentum $k_{F}$ for five different Skyrme parameterizations SLy4, SLy5, BSk19, BSk20, and BSk21.

\section{NUMERICAL RESULTS}

Let us now proceed as in Ref. 17 to compute the effect of the exchange of RPA excitations on pairing. We first compute the modified pairing interaction [Eqs. (2), (5), and (6)]. The summations and integrations in Eqs. (5) and (6) are all done numerically. The resulting matrix elements $V\left(k, k^{\prime}\right)$ are then used in the gap equation (1) to compute the critical temperature or the gap. Here, we will discuss $T_{c}$, from which the gap can be obtained to very good precision from $\Delta_{k_{F}}(T=0) \approx 1.76 T_{c}$ [27.

In our preceding work [17, using the Landau approximation, we found that there are strong cancellations between the attractive exchange of density fluctuations $(S=0)$ and the repulsive exchange of spin fluctuations $(S=1)$. While at low density, the repulsive effect was dominant, it turned out that at higher densities, the $S=0$ contribution became dominant due to its enhancement by the negative Landau parameter $f_{0}$ and the suppression of the $S=1$ contribution by the positive Landau parameter $g_{0}$.

Let us see how this picture is modified when one includes the full Skyrme RPA instead of the Landau approximation. In Fig. 5, we display a comparison of the critical temperatures obtained with different levels of ap-

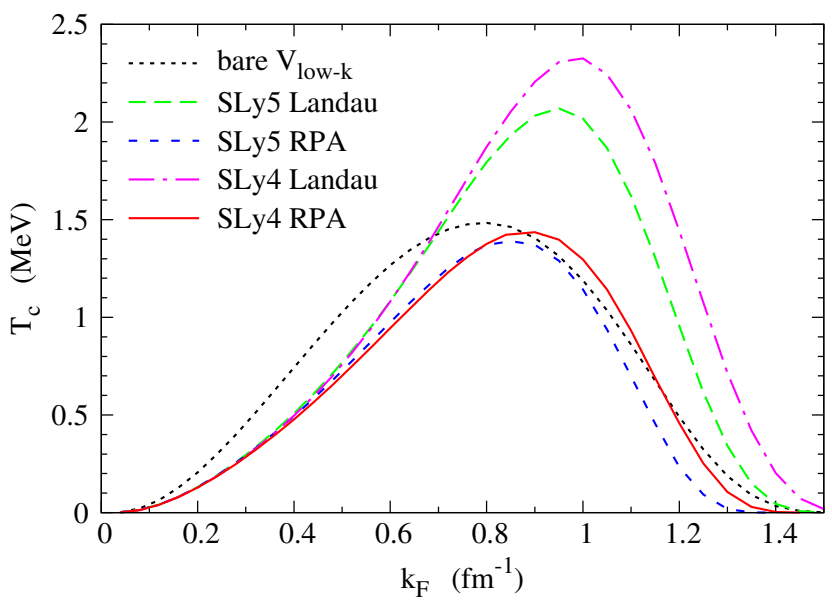

FIG. 5. Critical temperature $T_{c}$ as a function of $k_{F}$, obtained with the bare $V_{\text {low } k}$ (cutoff $\Lambda=\min \left(2.5 k_{F}, 2 \mathrm{fm}^{-1}\right.$ ), $m^{*}$ computed with SLy5) and with medium polarization calculated either within the Landau approximation or within the full RPA, with two different parameterizations of the Skyrme interaction (SLy4 and SLy5).

proximation. The black dotted curve represents the result without medium polarization. The long green dashes include the medium polarization computed within the Landau approximation using the SLy5 interaction. One clearly sees the suppression of $T_{c}$ at low density due to screening, gradually turning into an enhancement due to anti-screening at $k_{F} \gtrsim 0.7 \mathrm{fm}^{-1}$. This curve is very similar to the result we obtained in our previous work [17, while the one obtained with SLy4 (purple dashed-dotted curve) shows even stronger anti-screening at high density because of the larger value of the $G_{0}$ Landau parameter (see discussion in Sec. IV). The short blue dashed (SLy5) and the red solid (SLy4) lines are the corresponding results obtained within the full RPA instead of the Landau approximation. We see that for $k_{F} \lesssim 0.4 \mathrm{fm}^{-1}$, the full RPA and the Landau approximation agree very well. But at higher densities, the critical temperature within full RPA is always lower than within the Landau approximation. In the case of SLy5, the full RPA never gives anti-screening (i.e., enhancement of $T_{c}$ compared to the bare $V_{\text {low } k}$ interaction) and in the case of SLy4, antiscreening survives only in some range of densities around $k_{F} \sim 1 \mathrm{fm}^{-1}$ and it is much weaker than within the Landau approximation.

Qualitatively, this result can be understood by looking at Fig. 3. One sees two effects acting in the same direction: First, within the full RPA, the density response is less enhanced than within the Landau approximation, and, second, the spin response is less suppressed or even enhanced compared to the free one. (Strictly speaking, because of the spin-orbit interaction, the spin of the ph excitation is not a good quantum number any more, but nevertheless the argument remains qualitatively valid.)

The question arises whether this is a specific property of the SLy interactions or whether similar results 


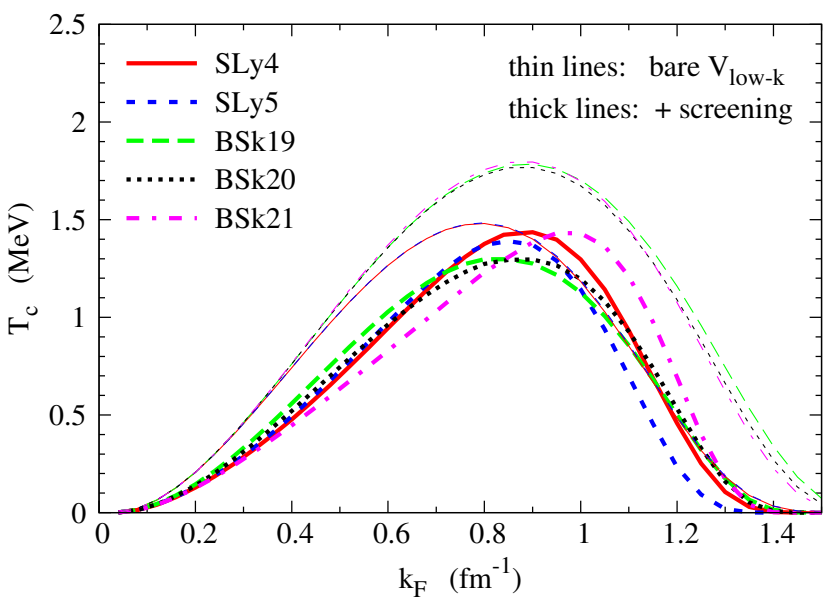

FIG. 6. Thin lines: critical temperature $T_{c}$ as a function of the Fermi momentum $k_{F}$, obtained with the the bare $V_{\text {low } k}$ [cutoff $\Lambda=\min \left(2.5 k_{F}, 2 \mathrm{fm}^{-1}\right)$ ] using the effective mass $m^{*}$ of the same Skyrme parameterizations as in Fig. 4. Thick lines: corresponding results including the medium polarization computed within full RPA.

are obtained with other Skyrme parameterizations. In Fig. 6. we compare the critical temperatures obtained with the bare $V_{\text {low } k}$ (thin lines) and with medium polarization within full RPA (thick lines) for five different Skyrme parameterizations (SLy4, SLy5, BSk19, BSk20, and BSk21). Looking at the thin lines, one sees that already at the level of the bare $V_{\text {low } k}$, the SLy and BSk parameterizations give quite different density dependence of $T_{c}$. This can easily be understood from Fig. 4 the SLy parameterizations predict a lower effective mass $m^{*}$ in neutron matter than the BSk ones. Since the gap and $T_{c}$ depend exponentially on the density of states $N_{0}=m^{*} k_{F} / \pi^{2}$, this has a dramatic effect, especially in the weak-coupling regime, i.e., at high density. Concerning the results with medium polarization (thick lines), none of the BSk forces gives anti-screening. At high densities, screening is strongest with BSk19 and weakest with BSk21, while at low densities, it is the opposite. There is a clear relationship between screening and the $G_{0}$ parameter: the more repulsive $G_{0}$ is, the weaker is the screening. This general trend is easily understood within the Landau approximation but apparently it also survives in the full RPA. A quite surprising result is that, at least if one compares these two families of Skyrme parameterizations, the model dependence with screening is weaker than without screening. Of course, this may be accidental.

In addition to the screening by the medium, there are correlations between neutrons above $T_{c}$ and such correlations can be taken into account via the Nozières-SchmittRink (NSR) approach [38, wherein, for a given chemical potential $\mu$, the density of the interacting neutrons is enhanced by the correlations calculated within the ladder approximation. As a result, the total density, $\rho_{\text {tot }}$ is writ-

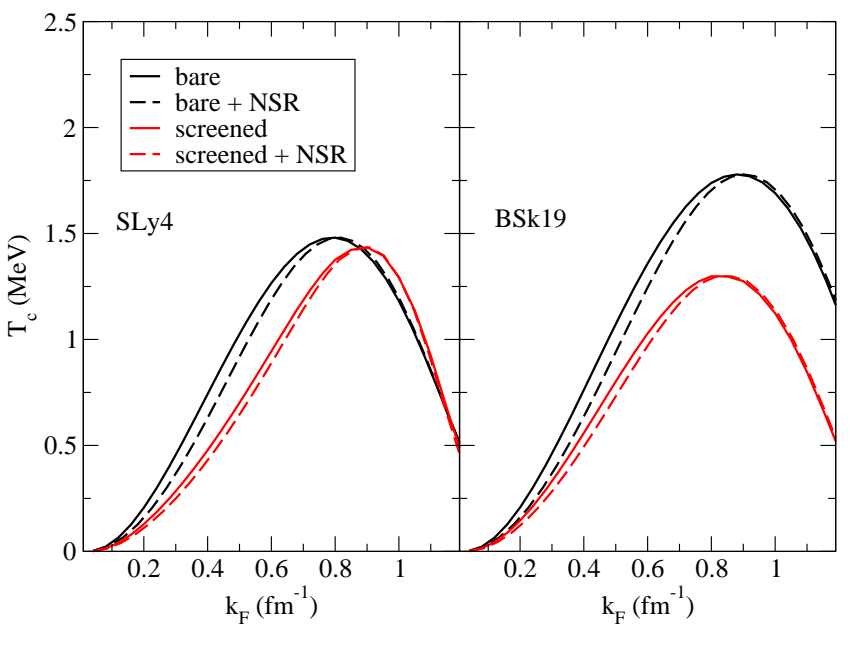

FIG. 7. Transition temperature versus $k_{F}$ or $k_{F, \text { tot }}$, respectively, obtained with (dashed lines) and without (solid lines) pair correlations via the Nozières-Schmitt-Rink procedure, both with the bare (black lines) and with the screened (red lines) interactions. All curves were obtained including the effective mass $m^{*}$ computed with the respective Skyrme interaction (SLy4 in the left panel and BSk19 in the right panel).

ten as a sum of uncorrelated and correlated densities,

$$
\rho_{\text {tot }}=\rho_{0}+\rho_{\text {corr }} .
$$

The formulas for $\rho_{0}$ and $\rho_{\text {corr }}$ are given in Appendix E. This effect is important at low densities where $T_{c} / \mu$ is not too small (strong coupling regime), as already seen in 17, 34. Since $T_{c}$ is computed according to the gap equation (1) as a function of $\mu$, but the relation between $\mu$ and $\rho_{\text {tot }}$ is changed, this implies that the dependence of $T_{c}$ on $\rho_{\text {tot }}$ (or on $k_{F, \text { tot }}=\left(3 \pi^{2} \rho_{\text {tot }}\right)^{1 / 3}$ ) is changed, too. Notice that now, for given $\rho_{\text {tot }}$ (or $k_{F, \text { tot }}$ ) the relation $\Delta(T=0)=1.76 T_{c}$, is no longer true.

In [17, we already studied the combined effect of correlations using the NSR approach, and screening in the Landau approximation. Since we have seen that the screening changes completely if one passes from the Landau approximation to the full Skyrme RPA, we would like to revisit this study using the Skyrme RPA instead of the Landau approximation.

Figure 7 shows the transition temperatures including the effect of preformed pairs via the NSR approach (i.e., as functions of $k_{F, \text { tot }}$ ), obtained with the bare and the screened interactions, respectively (black and red dashed lines). For ease of comparison, the figure also displays the transition temperatures calculated from the bare and the screened interaction without the NSR effect (i.e., as functions of $k_{F}$; black and red solid lines). All curves take into account the effective mass, which has been calculated using the respective Skyrme parameterizations (SLy4 in the left panel and BSk19 in the right panel).

As already observed in [17, we note that the effect of screening is much stronger than the effect of pair correlations, except at very low densities $\left(k_{F} \lesssim 0.1 \mathrm{fm}^{-1}\right.$ 
in the case of SLy4 and $k_{F} \lesssim 0.2 \mathrm{fm}^{-1}$ in the case of BSk19), where both effects are equally important (cf. black dashed and red solid lines).

As expected, the effect of preformed pairs is in fact limited to the range $k_{F} \lesssim 0.8 \mathrm{fm}^{-1}$, where the ratio $T_{c} / \mu$ is not too small so that the neutron matter is close to the socalled BCS-BEC crossover regime [39, 40]. This regime has been extensively studied with ultracold atoms 41] and in that case it was shown in Ref. 42 that by including simultaneously the effects of screening and pairing fluctuations (corresponding to the non-condensed preformed pairs), one reproduces very well the experimental result for the critical temperature in the unitary limit, i.e., in the case of a contact interaction with infinite scattering length.

In the present case of neutron matter, if the effect of pair correlations is included on top of screening (red dashed lines in Fig. 7), it is even weaker than in the case without screening, i.e., the difference between the solid and dashed red curves is smaller than the difference between the solid and dashed black curves, which do not include screening. This is because the screening weakens the attractive interaction, reducing the pairing correlations and hence the correlated density compared to the one obtained with the bare interaction, in agreement with our conclusions in our previous work (see Fig. 15 in [17).

\section{CONCLUSION}

The main goal of this work is to check the Landau approximation used in our previous work [17. (and in other recent studies of medium polarization effects 21-24) for the ph interaction while calculating diagram (b) seen in Fig. 1. To that end, we use the Skyrme interaction as it allows for easy computation of the RPA diagrams. For consistency, the same Skyrme interaction is also used in the calculation of the effective mass. We compare different parameterizations of the Saclay-Lyon family and of the more recent Brussels-Montreal family of Skyrme interactions. For the $3 \mathrm{p} 1 \mathrm{~h}$ and $1 \mathrm{p} 3 \mathrm{~h}$ vertices, we use the $V_{\text {low } k}$ interaction at a cutoff $\Lambda=\min \left(2.5 k_{F}, 2 \mathrm{fm}^{-1}\right)$, which is also used as bare pp interaction. As noted in [17, with the variable cutoff one can correctly account for the screening at low densities (GMB limit).

With the BSk and SLy families of interaction, there is model dependence already at the level of the bare $T_{c}$ due to differences in the effective mass. Surprisingly, the model dependence is reduced for the screened $T_{c}$, computed with the full RPA. Comparing the full RPA results with those of the Landau approximation, one finds that the Landau approximation is only valid at very low densities $\left(k_{F} \lesssim 0.4 \mathrm{fm}^{-1}\right.$, corresponding to $\left.\rho \lesssim 0.002 \mathrm{fm}^{-3}\right)$. At higher densities, screening is stronger i.e., $T_{c}$ is lower, with the full RPA. In particular, the anti-screening observed with the Landau approximation in [17] is completely lost, except with SLy4 in a small range of densities. In addition, one observes a correlation between the repulsion in the Landau parameter $G_{0}$ and the extent of screening, i.e., the more repulsive $G_{0}$ gets, the less screened is the dressed interaction. Qualitatively, this is easily understood in the Landau approximation, but it is interesting that this correlation is still present with the full RPA.

In this paper, we also include the correlations due to the preformed pairs via the NSR approach. While the effect from the preformed pairs is less drastic compared to the inclusion of medium polarization, we note that the correlations within NSR show up at low densities, where screening is strong even in the Landau approximation. As a result, the NSR effect of correlations on the density is weaker once screening is included.

While the transition temperatures at low density (say, up to $k_{F} \simeq 0.9 \mathrm{fm}^{-1}$, corresponding to $\sim 1 / 7$ of saturation density) are relatively robust, the high-density region is very sensitive to the approximation used. As noted here, momentum dependence of the ph interaction (and probably also of the effective mass, which is constant in the case of Skyrme interactions) seems to be a crucial ingredient in the extent to which the bare interaction is dressed. Perhaps techniques that allow for the building of the correlations from the medium, such as, inmedium similarity renormalization group might help in understanding the high-density region. Such a direction is crucial especially for a realistic description of pairing in the triplet channel.

\section{ACKNOWLEDGMENTS}

We would like to thank D. Davesne and A. Pastore for useful discussions about RPA response functions.

\section{Appendix A: Particle-hole interaction from Skyrme forces of the BSk family}

The energy functional corresponding to the generalized Skyrme force given in Eqs. (1) and (5) of Ref. 43 can be found in Appendix A of Ref. [37. Let us write it down (correcting a typo in the $C^{\nabla s}$ term) for the case of pure neutron matter:

$$
\begin{array}{r}
\mathcal{E}_{\mathrm{Sk}}=C^{\rho} \rho^{2}+C^{\tau}\left(\rho \tau-\mathbf{j}^{2}\right)+C^{\nabla \rho}(\boldsymbol{\nabla} \rho)^{2}+C^{s} \mathbf{s}^{2} \\
+C^{T}\left(\mathbf{s} \cdot \mathbf{T}-\mathbb{J}^{2}\right)+C^{\Delta s} \mathbf{s} \cdot \Delta \mathbf{s}+C^{\nabla s}(\boldsymbol{\nabla} \otimes \mathbf{s})^{2} \\
+C^{\nabla J}(\rho \boldsymbol{\nabla} \cdot \mathbf{J}+\mathbf{s} \cdot \boldsymbol{\nabla} \times \mathbf{j}) .
\end{array}
$$

For the definitions of the quantities $\rho, \tau, \mathbf{j}, \mathbf{s}, \mathbf{T}, \mathbb{J}$, and $\mathbf{J}$, see Ref. 35. Since we are dealing with pure neutron matter, the coefficients $C^{i}$ are the sum of isoscalar and isovector coefficients: $C^{i}=C_{0}^{i}+C_{1}^{i}$. While in standard Skyrme interactions only $C^{\rho}$ and $C^{s}$ are density dependent, this is now the case for all the $C^{i}$ except $C^{\nabla J}$. In Eq. A1, we have combined the $\rho \Delta \rho$ term of Ref. 37 with the $(\nabla \rho)^{2}$ term using integration by parts. Hence, 
in terms of the coefficients of Ref. 37, our coefficient $C^{\nabla \rho}$ corresponds to $C^{\nabla \rho}-\left(\rho C^{\Delta \rho}\right)^{\prime}$, where $X^{\prime}=d X / d \rho$. In contrast, the $\mathbf{s} \Delta \mathbf{s}$ term cannot be completely absorbed in the $(\boldsymbol{\nabla} \otimes \mathbf{s})^{2}$ term (or vice versa) because of the density dependence of $C^{\Delta s}$ and $C^{\nabla s}$, and we therefore keep both terms. Using the abbreviations

$$
\begin{aligned}
& \tilde{t}_{0}=t_{0}\left(1-x_{0}\right), \quad \tilde{t}_{1}=t_{1}\left(1-x_{1}\right), \quad \tilde{t}_{2}=t_{2}\left(1+x_{2}\right), \\
& \tilde{t}_{3}=t_{3}\left(1-x_{3}\right), \quad \tilde{t}_{4}=t_{4}\left(1-x_{4}\right), \quad \tilde{t}_{5}=t_{5}\left(1+x_{5}\right) \text {, }
\end{aligned}
$$

the coefficients $C^{i}$ can be written as

$$
\begin{aligned}
C^{\rho} & =\frac{1}{4} \tilde{t}_{0}+\frac{1}{24} \tilde{t}_{3} \rho^{\alpha}, \\
C^{\tau} & =\frac{1}{8}\left(\tilde{t}_{1}+3 \tilde{t}_{2}+\tilde{t}_{4} \rho^{\beta}+3 \tilde{t}_{5} \rho^{\gamma}\right), \\
C^{\nabla \rho} & =\frac{3}{32}\left(\tilde{t}_{1}-\tilde{t}_{2}+\left(1+\frac{2}{3} \beta\right) \tilde{t}_{4} \rho^{\beta}-\tilde{t}_{5} \rho^{\gamma}\right), \\
C^{s} & =-\frac{1}{4} \tilde{t}_{0}-\frac{1}{24} \tilde{t}_{3} \rho^{\alpha}, \\
C^{T} & =\eta_{J} \frac{1}{8}\left(-\tilde{t}_{1}+\tilde{t}_{2}-\tilde{t}_{4} \rho^{\beta}+\tilde{t}_{5} \rho^{\gamma}\right), \\
C^{\Delta s} & =\frac{1}{32}\left(3 \tilde{t}_{1}+\tilde{t}_{2}+2 \tilde{t}_{4} \rho^{\beta}\right), \\
C^{\nabla s} & =-\frac{1}{32}\left(\tilde{t}_{4} \rho^{\beta}+\tilde{t}_{5} \rho^{\gamma}\right), \\
C^{\nabla J} & =-W_{0} .
\end{aligned}
$$

For interactions which were fitted without the $\mathbb{J}^{2}$ terms (e.g., SLy4 25, BSk19-21 26]) one should use $\eta_{J}=0$, otherwise $\eta_{J}=1$ (e.g., for SLy5 [25]).

Inserting the functional (A1) into Eq. (8), one obtains the particle-hole interaction. In the case of the BSk interactions, the additional density dependence of $C^{\tau}$ leads to a slightly more general form than Eq. (11). To be specific, instead of one coefficient $v_{2}^{0}$, one needs now two independent coefficients $v_{2}^{0}$ and $v_{3}^{0}$ analogous to Eq. (14):

$$
\begin{gathered}
v_{1}^{0}(q)=\left(\rho^{2} C^{\rho}\right)^{\prime \prime}+\left(\rho C^{\tau}\right)^{\prime \prime} \tau+\left[2 C^{\nabla \rho}-\frac{1}{2}\left(\rho C^{\tau}\right)^{\prime}\right] q^{2}, \\
v_{2}^{0}=\left(\rho C^{\tau}\right)^{\prime}, \quad v_{3}^{0}=-2 C^{\tau}, \\
v_{4}^{0}(q)=2 C^{s}-2\left(C^{\Delta s}-C^{\nabla s}+\frac{1}{4} C^{s T}\right) q^{2}, \\
v_{5}^{0}=C^{s T}, \quad v_{6}^{0}=-2 C^{s T}, \quad v_{8}^{0}=v_{9}^{0}=C^{\nabla J},
\end{gathered}
$$

with $\tau=3 \rho k_{F}^{2} / 5$. All other $v_{i}^{0}$ are zero. The expressions for the $v_{i}^{0}$ in terms of the Skyrme parameters $\tilde{t}_{i}$ are readily obtained by inserting Eqs. $\mathrm{A} 3$ into Eqs. A4). They can also be obtained from the $\bar{W}_{i}^{(S)}$ of Ref. 37]: $v_{1}^{0}=\bar{W}_{1}^{(0)} / 2-\bar{W}_{3}^{(0)} q^{2} / 4, v_{2}^{0}=\bar{W}_{2}^{(0)} / 2$, $v_{3}^{0}=\bar{W}_{3}^{(0)}-\bar{W}_{2}^{(0)}, v_{4}^{0}=\bar{W}_{1}^{(1)} / 2-\bar{W}_{3}^{(1)} q^{2} / 4, v_{5}^{0}=\bar{W}_{2}^{(1)} / 2$, and $v_{6}^{0}=\bar{W}_{3}^{(1)}-\bar{W}_{2}^{(1)}$.

\section{Appendix B: Fermi liquid parameters}

From the ph interaction, it is straight forward to get the effective mass $m^{*}$ and the lowest-order Landau pa- rameters:

$$
\begin{aligned}
\frac{1}{m^{*}} & =\frac{1}{m}+2 \rho C^{\tau} . \\
f_{0} & =v_{1}^{0}(q=0)+2 v_{2}^{0} k_{F}^{2} \\
& =\left(\rho^{2} C^{\rho}\right)^{\prime \prime}+\left[\frac{3}{5} \rho\left(\rho C^{\tau}\right)^{\prime \prime}+2\left(\rho C^{\tau}\right)^{\prime}\right] k_{F}^{2}, \\
g_{0} & =v_{4}^{0}(q=0)+2 v_{5}^{0} k_{F}^{2}=2 C^{s}+2 C^{s T} k_{F}^{2} .
\end{aligned}
$$

The dimensionless Landau parameters $F_{0}$ and $G_{0}$ shown in Fig. 4 are defined as $F_{0}=N_{0} f_{0}$ and $G_{0}=N_{0} g_{0}$. Our expressions (B1), (B2), and (B3) for the Fermi-liquid parameters coincide with those given in Eqs. (B3), (B1a), and (B1c) of Ref. [26].

\section{Appendix C: Generalized Lindhard functions}

The generalized Lindhard functions $\Pi_{n}$ can be computed analytically. It is convenient to write them as $\Pi_{n}=N_{0} k_{F}^{n} \tilde{\Pi}_{n}$, where $\tilde{\Pi}_{n}$ are dimensionless functions of $\tilde{q}=q / k_{F}$ :

$$
\begin{aligned}
\tilde{\Pi}_{0} & =-\frac{1}{2}-\frac{4-\tilde{q}^{2}}{8 \tilde{q}} \ln \left|\frac{2+\tilde{q}}{2-\tilde{q}}\right| \\
\tilde{\Pi}_{2} & =-\frac{12-\tilde{q}^{2}}{16}-\frac{\left(4-\tilde{q}^{2}\right)^{2}}{64 \tilde{q}} \ln \left|\frac{2+\tilde{q}}{2-\tilde{q}}\right| \\
\tilde{\Pi}_{2 L} & =-\frac{1}{3} \\
\tilde{\Pi}_{4} & =-\frac{240-8 \tilde{q}^{2}+3 \tilde{q}^{4}}{288}-\frac{\left(4-\tilde{q}^{2}\right)^{3}}{384 \tilde{q}} \ln \left|\frac{2+\tilde{q}}{2-\tilde{q}}\right| .
\end{aligned}
$$

The function $\Pi_{2 T}$ can be obtained from $\Pi_{2}$ and $\Pi_{2 L}$ according to Eq. [17).

Notice that our functions $\Pi_{n}$ are defined differently from those in Refs. 30, 31.

\section{Appendix D: Solution of the RPA equation}

The Skyrme interaction $\mathcal{V}^{0}$ can be written in a form analogous to Eq. (14) (similar to Eq. (11) but generalized to $v_{3}^{0} \neq-2 v_{2}^{0}$ in the case of BSk interactions, see Appendix A, with the non-vanishing coefficients $v_{i}^{0}$ given in Eqs. A4 and all other $v_{i}^{0}=0$. Inserting this and Eq. (14) into Eq. (12), one gets 


$$
\begin{aligned}
& \mathcal{V}_{21}=\mathcal{V}_{21}^{0} \\
& +\left\{v_{1}^{0} \Pi_{0}+v_{2}^{0}\left[p_{2}^{2} \Pi_{0}+\Pi_{2}\right]-v_{8}^{0} i \mathbf{q} \cdot \mathbf{p}_{2} \times \boldsymbol{\sigma}_{2} \Pi_{0}\right\} v_{1} \\
& +\left\{v_{1}^{0}\left[p_{1}^{2} \Pi_{0}+\Pi_{2}\right]+v_{2}^{0}\left[p_{1}^{2} p_{2}^{2} \Pi_{0}+\left(p_{1}^{2}+p_{2}^{2}\right) \Pi_{2}+\Pi_{4}\right]-v_{8}^{0} i \mathbf{q} \cdot \mathbf{p}_{2} \times \boldsymbol{\sigma}_{2}\left[p_{1}^{2} \Pi_{0}+\Pi_{2}\right]\right\} v_{2} \\
& +\left\{v_{3}^{0}\left[\mathbf{p}_{1} \cdot \mathbf{p}_{2} \Pi_{2 T}+\mathbf{p}_{1} \cdot \mathbf{q} \mathbf{p}_{2} \cdot \mathbf{q}\left(\Pi_{2 L}-\Pi_{2 T}\right) / q^{2}\right]+v_{9}^{0} i \mathbf{q} \cdot \mathbf{p}_{1} \times \boldsymbol{\sigma}_{2} \Pi_{2 T}\right\} v_{3} \\
& +\left\{v_{4}^{0} \boldsymbol{\sigma}_{1} \cdot \boldsymbol{\sigma}_{2} \Pi_{0}+v_{5}^{0} \boldsymbol{\sigma}_{1} \cdot \boldsymbol{\sigma}_{2}\left[p_{2}^{2} \Pi_{0}+\Pi_{2}\right]-v_{9}^{0} i \mathbf{q} \cdot \mathbf{p}_{2} \times \boldsymbol{\sigma}_{1} \Pi_{0}\right\} v_{4} \\
& +\left\{v_{4}^{0} \boldsymbol{\sigma}_{1} \cdot \boldsymbol{\sigma}_{2}\left[p_{1}^{2} \Pi_{0}+\Pi_{2}\right]+v_{5}^{0} \boldsymbol{\sigma}_{1} \cdot \boldsymbol{\sigma}_{2}\left[p_{1}^{2} p_{2}^{2} \Pi_{0}+\left(p_{1}^{2}+p_{2}^{2}\right) \Pi_{2}+\Pi_{4}\right]-v_{9}^{0} i \mathbf{q} \cdot \mathbf{p}_{2} \times \boldsymbol{\sigma}_{1}\left[p_{1}^{2} \Pi_{0}+\Pi_{2}\right]\right\} v_{5} \\
& +\left\{v_{6}^{0} \boldsymbol{\sigma}_{1} \cdot \boldsymbol{\sigma}_{2}\left[\mathbf{p}_{1} \cdot \mathbf{p}_{2} \Pi_{2 T}+\mathbf{p}_{1} \cdot \mathbf{q} \mathbf{p}_{2} \cdot \mathbf{q}\left(\Pi_{2 L}-\Pi_{2 T}\right) / q^{2}\right]+v_{8}^{0} i \mathbf{q} \cdot \mathbf{p}_{1} \times \boldsymbol{\sigma}_{1} \Pi_{2 T}\right\} v_{6} \\
& +\left\{v_{4}^{0} \boldsymbol{\sigma}_{1} \cdot \mathbf{q} \boldsymbol{\sigma}_{2} \cdot \mathbf{q} \Pi_{0}+v_{5}^{0} \boldsymbol{\sigma}_{1} \cdot \mathbf{q} \boldsymbol{\sigma}_{2} \cdot \mathbf{q}\left[p_{2}^{2} \Pi_{0}+\Pi_{2}\right]\right\} v_{7} \\
& +\left\{v_{1}^{0} i \mathbf{q} \cdot \mathbf{p}_{1} \times \boldsymbol{\sigma}_{1} \Pi_{0}+v_{2}^{0} i \mathbf{q} \cdot \mathbf{p}_{1} \times \boldsymbol{\sigma}_{1}\left[p_{2}^{2} \Pi_{0}+\Pi_{2}\right]-v_{6}^{0} i \mathbf{q} \cdot \mathbf{p}_{2} \times \boldsymbol{\sigma}_{2} \Pi_{2 T}+v_{8}^{0}\left[\mathbf{q} \cdot \mathbf{p}_{1} \times \boldsymbol{\sigma}_{1} \mathbf{q} \cdot \mathbf{p}_{2} \times \boldsymbol{\sigma}_{2} \Pi_{0}+2 q^{2} \Pi_{2 T}\right]\right\} v_{8} \\
& +\left\{-v_{3}^{0} i \mathbf{q} \cdot \mathbf{p}_{2} \times \boldsymbol{\sigma}_{1} \Pi_{2 T}+v_{4}^{0} i \mathbf{q} \cdot \mathbf{p}_{1} \times \boldsymbol{\sigma}_{2} \Pi_{0}+v_{5}^{0} i \mathbf{q} \cdot \mathbf{p}_{1} \times \boldsymbol{\sigma}_{2}\left[p_{2}^{2} \Pi_{0}+\Pi_{2}\right]\right. \\
& \left.+v_{9}^{0}\left[\left(\mathbf{p}_{1} \cdot \mathbf{p}_{2} q^{2}-\mathbf{p}_{1} \cdot \mathbf{q} \mathbf{p}_{2} \cdot \mathbf{q}\right) \Pi_{0}+\left(\boldsymbol{\sigma}_{1} \cdot \boldsymbol{\sigma}_{2} q^{2}-\boldsymbol{\sigma}_{1} \cdot \mathbf{q} \boldsymbol{\sigma}_{2} \cdot \mathbf{q}\right) \Pi_{2 T}\right]\right\} v_{9} \\
& +\left\{v_{1}^{0} p_{1}^{2} \Pi_{2}+v_{2}^{0} p_{1}^{2}\left[p_{2}^{2} \Pi_{2}+\Pi_{4}\right]-v_{8}^{0} i \mathbf{q} \cdot \mathbf{p}_{2} \times \boldsymbol{\sigma}_{2} p_{1}^{2} \Pi_{2}\right\} v_{10} \\
& +v_{3}^{0} \mathbf{p}_{1} \cdot \mathbf{q} \mathbf{p}_{2} \cdot \mathbf{q} \Pi_{2 L} v_{11} \\
& +\left\{v_{4}^{0} \boldsymbol{\sigma}_{1} \cdot \boldsymbol{\sigma}_{2} p_{1}^{2} \Pi_{2}+v_{5}^{0} \boldsymbol{\sigma}_{1} \cdot \boldsymbol{\sigma}_{2} p_{1}^{2}\left[p_{2}^{2} \Pi_{2}+\Pi_{4}\right]-v_{9}^{0} i \mathbf{q} \cdot \mathbf{p}_{2} \times \boldsymbol{\sigma}_{1} p_{1}^{2} \Pi_{2}\right\} v_{12} \\
& +v_{6}^{0} \boldsymbol{\sigma}_{1} \cdot \boldsymbol{\sigma}_{2} \mathbf{p}_{1} \cdot \mathbf{q} \mathbf{p}_{2} \cdot \mathbf{q} \Pi_{2 L} v_{13} \\
& +\left\{v_{4}^{0} \boldsymbol{\sigma}_{1} \cdot \mathbf{q} \boldsymbol{\sigma}_{2} \cdot \mathbf{q}\left[p_{1}^{2} \Pi_{0}+\Pi_{2}\right]+v_{5}^{0} \boldsymbol{\sigma}_{1} \cdot \mathbf{q} \boldsymbol{\sigma}_{2} \cdot \mathbf{q}\left[p_{1}^{2} p_{2}^{2} \Pi_{0}+\left(p_{1}^{2}+p_{2}^{2}\right) \Pi_{2}+\Pi_{4}\right]\right\} v_{14} \\
& +\left\{v_{4}^{0} \boldsymbol{\sigma}_{1} \cdot \mathbf{q} \boldsymbol{\sigma}_{2} \cdot \mathbf{q} p_{1}^{2} \Pi_{2}+v_{5}^{0} \boldsymbol{\sigma}_{1} \cdot \mathbf{q} \boldsymbol{\sigma}_{2} \cdot \mathbf{q} p_{1}^{2}\left[p_{2}^{2} \Pi_{2}+\Pi_{4}\right]\right\} v_{15} \\
& +\left\{v_{1}^{0} i \mathbf{q} \cdot \mathbf{p}_{1} \times \boldsymbol{\sigma}_{1} \Pi_{2}+v_{2}^{0} i \mathbf{q} \cdot \mathbf{p}_{1} \times \boldsymbol{\sigma}_{1}\left[p_{2}^{2} \Pi_{2}+\Pi_{4}\right]-v_{6}^{0} i \mathbf{q} \cdot \mathbf{p}_{2} \times \boldsymbol{\sigma}_{2} p_{1}^{2} \Pi_{2 T}\right. \\
& \left.+v_{8}^{0}\left[\mathbf{q} \cdot \mathbf{p}_{1} \times \boldsymbol{\sigma}_{1} \mathbf{q} \cdot \mathbf{p}_{2} \times \boldsymbol{\sigma}_{2} \Pi_{2}+2 q^{2} p_{1}^{2} \Pi_{2 T}\right]\right\} v_{16} \\
& +\left\{-v_{3}^{0} i \mathbf{q} \cdot \mathbf{p}_{2} \times \boldsymbol{\sigma}_{1} p_{1}^{2} \Pi_{2 T}+v_{4}^{0} i \mathbf{q} \cdot \mathbf{p}_{1} \times \boldsymbol{\sigma}_{2} \Pi_{2}+v_{5}^{0} i \mathbf{q} \cdot \mathbf{p}_{1} \times \boldsymbol{\sigma}_{2}\left[p_{2}^{2} \Pi_{2}+\Pi_{4}\right]\right. \\
& \left.+v_{9}^{0}\left[\left(\mathbf{p}_{1} \cdot \mathbf{p}_{2} q^{2}-\mathbf{p}_{1} \cdot \mathbf{q} \mathbf{p}_{2} \cdot \mathbf{q}\right) \Pi_{2}+\left(\boldsymbol{\sigma}_{1} \cdot \boldsymbol{\sigma}_{2} q^{2}-\boldsymbol{\sigma}_{1} \cdot \mathbf{q} \boldsymbol{\sigma}_{2} \cdot \mathbf{q}\right) p_{1}^{2} \Pi_{2 T}\right]\right\} v_{17} \\
& +\left\{v_{6}^{0} \mathbf{q} \cdot \mathbf{p}_{1} \times \boldsymbol{\sigma}_{1} \mathbf{q} \cdot \mathbf{p}_{2} \times \boldsymbol{\sigma}_{2} \Pi_{2 T}+v_{8}^{0} i \mathbf{q} \cdot \mathbf{p}_{1} \times \boldsymbol{\sigma}_{1} 2 q^{2} \Pi_{2 T}\right\} v_{18} \text {. }
\end{aligned}
$$

By collecting the coefficients of the different operators, we obtain a system of linear equations for the unknown $v_{i}$, of the form (18).

Notice that for some of the $v_{i}$, the equations are not unique. For instance, the equation for $v_{2}$ can be obtained from the coefficients of $p_{1}^{2}$ or $p_{2}^{2}$. However, the final result is independent of this choice because the equality of the coefficients follows from the hermiticity of $V_{12}=V_{21}^{*}$. Here, we choose the equations for $v_{2}, v_{5}, v_{8}, v_{9}, v_{14}$, $v_{16}$, and $v_{17}$ that are obtained from the coefficients of $p_{2}^{2}, \boldsymbol{\sigma}_{1} \cdot \boldsymbol{\sigma}_{2} p_{2}^{2},-i \mathbf{q} \cdot \mathbf{p}_{2} \times \boldsymbol{\sigma}_{2},-i \mathbf{q} \cdot \mathbf{p}_{2} \times \boldsymbol{\sigma}_{1}, \boldsymbol{\sigma}_{1} \cdot \mathbf{q} \boldsymbol{\sigma}_{2} \cdot \mathbf{q} p_{2}^{2}$, $i \mathbf{q} \cdot \mathbf{p}_{1} \times \boldsymbol{\sigma}_{1} p_{2}^{2}$, and $i \mathbf{q} \cdot \mathbf{p}_{1} \times \boldsymbol{\sigma}_{2} p_{2}^{2}$, respectively.

With this choice, the non-vanishing matrix elements in Eq. 18 are:

$$
\begin{gathered}
A_{1,1}=v_{1}^{0} \Pi_{0}+v_{2}^{0} \Pi_{2}, \quad A_{1,2}=v_{1}^{0} \Pi_{2}+v_{2}^{0} \Pi_{4}, \\
A_{1,8}=2 v_{8}^{0} q^{2} \Pi_{2 T}, \quad A_{2,1}=v_{2}^{0} \Pi_{0}, \quad A_{2,2}=v_{2}^{0} \Pi_{2}, \\
A_{3,3}=v_{3}^{0} \Pi_{2 T}, \quad A_{3,9}=v_{9}^{0} q^{2} \Pi_{0}, \quad A_{3,17}=v_{9}^{0} q^{2} \Pi_{2}, \\
A_{4,4}=v_{4}^{0} \Pi_{0}+v_{5}^{0} \Pi_{2}, \quad A_{4,5}=v_{4}^{0} \Pi_{2}+v_{5}^{0} \Pi_{4}, \\
A_{4,9}=v_{9}^{0} q^{2} \Pi_{2 T}, \quad A_{5,4}=v_{5}^{0} \Pi_{0}, \quad A_{5,5}=v_{5}^{0} \Pi_{2}, \\
A_{6,6}=v_{6}^{0} \Pi_{2 T}, \quad A_{7,7}=v_{4}^{0} \Pi_{0}+v_{5}^{0} \Pi_{2},
\end{gathered}
$$

$$
\begin{gathered}
A_{7,9}=-v_{9}^{0} \Pi_{2 T}, \quad A_{7,14}=v_{4}^{0} \Pi_{2}+v_{5}^{0} \Pi_{4}, \\
A_{8,1}=v_{8}^{0} \Pi_{0}, \quad A_{8,2}=v_{8}^{0} \Pi_{2}, \quad A_{8,8}=v_{6}^{0} \Pi_{2 T}, \\
A_{9,4}=v_{9}^{0} \Pi_{0}, \quad A_{9,5}=v_{9}^{0} \Pi_{2}, \quad A_{9,9}=v_{3}^{0} \Pi_{2 T}, \\
A_{10,2}=v_{2}^{0} \Pi_{0}, \quad A_{10,10}=v_{2}^{0} \Pi_{2}, \\
A_{11,3}=v_{3}^{0}\left(\Pi_{2 L}-\Pi_{2 T}\right) / q^{2}, \quad A_{11,9}=-v_{9}^{0} \Pi_{0}, \\
A_{11,11}=v_{3}^{0} \Pi_{2 L}, \quad A_{11,17}=-v_{9}^{0} \Pi_{2}, \quad A_{12,5}=v_{5}^{0} \Pi_{0}, \\
A_{12,12}=v_{5}^{0} \Pi_{2}, \quad A_{13,6}=v_{6}^{0}\left(\Pi_{2 L}-\Pi_{2 T}\right) / q^{2}, \\
A_{13,13}=v_{6}^{0} \Pi_{2 L}, \quad A_{14,7}=v_{5}^{0} \Pi_{0}, \quad A_{14,14}=v_{5}^{0} \Pi_{2}, \\
A_{15,14}=v_{5}^{0} \Pi_{0}, \quad A_{15,15}=v_{5}^{0} \Pi_{2}, \quad A_{16,8}=v_{2}^{0} \Pi_{0}, \\
A_{16,16}=v_{2}^{0} \Pi_{2}, \quad A_{17,9}=v_{5}^{0} \Pi_{0}, \quad A_{17,17}=v_{5}^{0} \Pi_{2}, \\
A_{18,8}=v_{8}^{0} \Pi_{0}, \quad A_{18,16}=v_{8}^{0} \Pi_{2}, \quad A_{18,18}=v_{6}^{0} \Pi_{2 T} .
\end{gathered}
$$

This system of equations has actually two decoupled blocks corresponding to the indices $1,2,6,8,10,13$, 16,18 , which have products of two time-even operators, and to the indices $3,4,5,7,9,11,12,14,15,17$, which have products of two time-odd operators. 
The solution for the time-even operators reads:

$$
\begin{gathered}
v_{1}=\frac{\phi_{a}-1}{\Pi_{0}}, \quad v_{2}=v_{2}^{0} \phi_{2} \phi_{a}, \quad v_{6}=v_{6}^{0} \phi_{6 T}, \\
v_{8}=v_{8}^{0} \phi_{2} \phi_{6 T} \phi_{a}, \quad v_{10}=\left(v_{2}^{0}\right)^{2} \Pi_{0} \phi_{2}^{2} \phi_{a}, \\
v_{13}=\frac{\left(v_{6}^{0}\right)^{2}\left(\Pi_{2 L}-\Pi_{2 T}\right) \phi_{6 L} \phi_{6 T}}{q^{2}} \\
v_{16}=v_{2}^{0} v_{8}^{0} \Pi_{0} \phi_{2}^{2} \phi_{6 T} \phi_{a}, \quad v_{18}=\left(v_{8}^{0}\right)^{2} \Pi_{0} \phi_{2}^{2} \phi_{6 T}^{2} \phi_{a},
\end{gathered}
$$

and the solution for the time-odd operators:

$$
\begin{gathered}
v_{3}=\frac{\phi_{3 T} \phi_{c}-1}{\Pi_{2 T}}, \quad v_{4}=\frac{\phi_{b} \phi_{c}-\phi_{5}^{2}}{\Pi_{0} \phi_{5}^{2}}, \quad v_{5}=\frac{v_{5}^{0} \phi_{b} \phi_{c}}{\phi_{5}}, \\
v_{7}=-\frac{\left(v_{9}^{0}\right)^{2} \Pi_{2 T} \phi_{3 T} \phi_{b}^{2} \phi_{c}}{\phi_{5}^{2}}, \quad v_{9}=\frac{v_{9}^{0} \phi_{3 T} \phi_{b} \phi_{c}}{\phi_{5}}, \\
v_{11}=\frac{\phi_{3 L}\left[1-\phi_{c}-\left(1-\phi_{3 T} \phi_{c}\right) v_{3}^{0}\left(\Pi_{2 L}-\Pi_{2 T}\right)\right]}{q^{2} \Pi_{2 T}}, \\
v_{12}=\left(v_{5}^{0}\right)^{2} \Pi_{0} \phi_{b} \phi_{c}, \quad v_{14}=-\frac{v_{5}^{0}\left(v_{9}^{0}\right)^{2} \Pi_{0} \Pi_{2 T} \phi_{3 T} \phi_{b}^{2} \phi_{c}}{\phi_{5}}, \\
v_{15}=-\left(v_{5}^{0} v_{9}^{0}\right)^{2} \Pi_{0}^{2} \Pi_{2 T} \phi_{3 T} \phi_{b}^{2} \phi_{c}, \\
v_{17}=v_{5}^{0} v_{9}^{0} \Pi_{0} \phi_{3 T} \phi_{b} \phi_{c},
\end{gathered}
$$

where the following abbreviations have been used:

$$
\begin{gathered}
\phi_{2}=\frac{1}{1-v_{2}^{0} \Pi_{2}}, \quad \phi_{3 L, T}=\frac{1}{1-v_{3}^{0} \Pi_{2 L, T}}, \\
\phi_{5}=\frac{1}{1-v_{5}^{0} \Pi_{2}}, \quad \phi_{6 L, T}=\frac{1}{1-v_{6}^{0} \Pi_{2 L, T}}, \\
\phi_{a}=\frac{1}{1-\Pi_{0}\left[v_{1}^{0}+\left(v_{2}^{0}\right)^{2} \Pi_{4}+2 q^{2}\left(v_{8}^{0}\right)^{2} \Pi_{2 T} \phi_{6 T}\right] \phi_{2}^{2}} \\
\phi_{b}=\frac{1}{1-v_{4}^{0} \Pi_{0}-2 v_{5}^{0} \Pi_{2}+\left(v_{5}^{0}\right)^{2}\left(\Pi_{2}^{2}-\Pi_{0} \Pi_{4}\right)}, \\
\phi_{c}=\frac{1}{1-q^{2}\left(v_{9}^{0}\right)^{2} \Pi_{0} \Pi_{2 T} \phi_{3 T} \phi_{b}} .
\end{gathered}
$$

\section{Appendix E: Correlated density (NSR correction)}

In this appendix we summarize the formulas needed for the calculation of the NSR correction. For their derivation and more details, see Refs. [17, 34]. For a given chemical potential $\mu$, the neutron density $\rho$ is written as a sum of three terms,

$$
\rho=\rho_{0}+\rho_{\mathrm{corr}, 1}+\rho_{\mathrm{corr}, 2} .
$$

The first term, $\rho_{0}$, is the uncorrelated density,

$$
\rho_{0}=2 \sum_{\mathbf{p}} f\left(\xi_{p}\right)
$$

where $f\left(\xi_{p}\right)=1 /\left(e^{\xi_{p} / T}+1\right)$ is the Fermi-Dirac distribution function, $\xi_{p}=\epsilon_{p}-\mu$, and the factor of 2 arises due to the spin degeneracy. The other terms are the correlated density calculated to first order in the singleparticle self-energy $\Sigma$. The term $\rho_{\text {corr }, 1}$ corresponds to the original NSR correction [38] and is given by

$$
\rho_{\mathrm{corr}, 1}=2 T \sum_{\mathbf{p}, \omega_{n}}\left[\mathcal{G}_{0}\left(\mathbf{p}, \omega_{n}\right)\right]^{2} \Sigma\left(\mathbf{p}, i \omega_{n}\right)
$$

where $\omega_{n}$ are the fermionic Matsubara frequencies and $\mathcal{G}_{0}=1 /\left(i \omega_{n}-\xi_{p}\right)$ is the uncorrelated single-particle Green's function in the imaginary-time formalism [27. Calculating $\Sigma\left(\mathbf{p}, i \omega_{n}\right)$ within the ladder approximation and performing a couple of steps detailed in [34], one finally obtains the expression

$$
\rho_{\text {corr }, 1}=-\frac{\partial}{\partial \mu} \sum_{\mathbf{K}, \nu} \int \frac{d \omega}{\pi} g(\omega) \operatorname{Im} \log \left[1-\eta_{\nu}(K, \omega)\right]
$$

Here, $g(\omega)=1 /\left(e^{\beta \omega}-1\right)$ is the Bose function and $\eta_{\nu}$ are the complex eigenvalues of

$$
V \bar{G}^{(2)}=V\left(k, k^{\prime}\right) \frac{\bar{Q}\left(K, k^{\prime}\right)}{\omega-\frac{K^{2}}{4 m^{*}}-\frac{k^{\prime 2}}{m^{*}}+2 \mu},
$$

where $\bar{Q}$ denotes the angle average (since we consider only the $s$ wave) of the Pauli blocking factor $Q(\mathbf{K}, \mathbf{k})=$ $1-f\left(\xi_{\frac{\mathbf{K}}{2}-\mathbf{k}}\right)-f\left(\xi_{\frac{\mathbf{K}}{2}+\mathbf{k}}\right)$ where $\mathbf{K}$ is the total momentum of the pair. In principle, the screening correction to $V\left(k, k^{\prime}\right)$ should also depend on $K$, but as shown in 17 this dependence is weak and we neglect it.

The last term, $\rho_{\text {corr }, 2}$ is absent in the original NSR approach. It takes into account that $\mathcal{G}_{0}$ includes already the in-medium quasiparticle energy $\xi_{p}$ which therefore must not be shifted by the self-energy [44, 45. Accordingly, one has to subtract from the self-energy in Eq. (E3) its on-shell value $\operatorname{Re} \Sigma\left(\mathbf{p}, \xi_{p}\right)$, which gives

$$
\rho_{\text {corr }, 2}=-2 T \sum_{\mathbf{p}, \omega_{n}}\left[\mathcal{G}_{0}\left(\mathbf{p}, \omega_{n}\right)\right]^{2} \operatorname{Re} \Sigma\left(\mathbf{p}, \xi_{p}\right)
$$

As in [17, 34, we approximate $\Sigma\left(\mathbf{p}, \xi_{p}\right)$ by the first-order (Hartree-Fock) self-energy and finally arrive at the following correction:

$$
\rho_{\mathrm{corr}, 2}=4 \pi \frac{\partial}{\partial \mu} \sum_{\mathbf{K}, \mathbf{k}} g\left(\frac{K^{2}}{4 m^{*}}+\frac{k^{2}}{m^{*}}-2 \mu\right) V(k, k) \bar{Q}(K, k) .
$$

[1] D. J. Dean and M. Hjorth-Jensen, Rev. Mod. Phys. 75 607 (2003).
[2] B. Haskell and A. Sedrakian, arXiv:1709.10340 [astro- 
ph.HE].

[3] A. Sedrakian and J. W. Clark, Eur. Phys. J. A 55, 167 (2019)

[4] A. B. Migdal, Zh. Eksp. Teor. Fiz. 37249 (1959) [Sov. Phys. JETP 10, 176 (1960)]; Nucl. Phys. 13, 655 (1959).

[5] V. L. Ginzburg and D. A. Kirzhnits, Zh. Eksp. Teor. Fiz. 47, 2006 (1964) [Sov. Phys. JETP 20, 1346 (1965)].

[6] D. G. Yakovlev and C. J. Pethick, Ann. Rev. Astron. Astrophys. 42, 169 (2004).

[7] D. Page, J. M. Lattimer, M. Prakash and A. W. Steiner, Astrophys. J. 707, 1131 (2009).

[8] P. W. Anderson and N. Itoh, Nature 256, 25 (1975).

[9] D. Pines and M. A. Alpar, Nature 316, 27 (1985).

[10] G. Baym, C. J. Pethick, D. Pines, and Malvin Ruderman, Nature 224, 872 (1969).

[11] N. Chamel and P. Haensel, Living Rev. Relativity, 11, 10 (2008).

[12] S. Maurizio, J. W. Holt and P. Finelli, Phys. Rev. C 90 044003 (2014).

[13] S. Srinivas and S. Ramanan, Phys. Rev. C 94, 064303 (2016).

[14] C. Drischler, T. Krüger, K. Hebeler and A. Schwenk, Phys. Rev. C 95, 024302 (2017).

[15] P. Papakonstantinou and J. W. Clark, J. Low. Temp. Phys. 189, 361 (2017).

[16] K. Hebeler, A. Schwenk, and B. Friman, Phys. Lett. B 648176 (2007).

[17] S. Ramanan and M. Urban, Phys. Rev. C 98, 024314 (2018).

[18] S. Gandolfi, A. Yu. Illarionov, S. Fantoni, F. Pederiva, and K. E. Schmidt, Phys. Rev. Lett. 101, 132501, (2008).

[19] T. Abe and R. Seki Phys. Rev. C 79, 054003 (2009).

[20] A. Gezerlis and J. Carlson, Phys. Rev. C 81, 025803 (2010).

[21] Caiwan Shen, U. Lombardo, and P. Schuck, Phys. Rev. C 71, 054301 (2005).

[22] L. G. Cao, U. Lombardo, and P. Schuck, Phys. Rev. C 74, 064301 (2006).

[23] D. Ding, A. Rios, H. Dussan, W. H. Dickhoff, S. J. Witte, A. Carbone, and A. Polls, Phys. Rev. C 94, 025802 (2016).

[24] S. S. Zhang, L. G. Cao, U. Lombardo, and P. Schuck, Phys. Rev. C 93, 044329 (2016).

[25] E. Chabanat, P. Bonche, P. Haensel, J. Meyer, and R.
Schaeffer, Nucl. Phys. A 635, 231 (1998).

[26] S. Goriely, N. Chamel, and J.M. Pearson, Phys. Rev. C 82, 035804 (2010).

[27] A. L. Fetter and J. D. Walecka, Quantum Theory of Many-Particle Systems (McGraw-Hill, New York, 1971).

[28] S. K. Bogner, R. J. Furnstahl, S. Ramanan, and A. Schwenk, Nucl. Phys. A 784, 79 (2007).

[29] D. A. Varshalovich, A. N. Moskalev, V. K. Khersonskii, Quantum Theory of Angular Momentum (World Scientific, Singapore, 1988).

[30] A. Pastore, D. Davesne, and J. Navarro, Phys. Rep. 563, 1 (2015).

[31] C. García-Recio, J. Navarro, Van Giai Nguyen, and L.L. Salcedo, Ann. Phys. (N.Y.) 214, 293 (1992).

[32] M. Buraczynski and A. Gezerlis, Phys. Rev. Lett. 116, 152501 (2016)

[33] L. P. Gor'kov and T.K. Melik-Barkhudarov, Zh. Eksp. Teor. Fiz. 40, 1452 (1961) [Sov. Phys. JETP 13, 1018 (1961)].

[34] S. Ramanan and M. Urban, Phys. Rev. C 88, 054315 (2013).

[35] M. Bender, P.-H. Heenen, and P.-G. Reinhard, Rev. Mod. Phys. 75, 121 (2003).

[36] N. Chamel and S. Goriely, Phys. Rev. C 82, 045804 (2010).

[37] A. Pastore, M. Martini, D. Davesne, J. Navarro, S. Goriely, and N. Chamel, Phys. Rev. C 90, 025804 (2014).

[38] P. Nozières and S. Schmitt-Rink, J. Low. Temp. Phys. 59, 195 (1985).

[39] M. Matsuo, Phys. Rev. C 73, 044309 (2006).

[40] J. Margueron, H. Sagawa, and K. Hagino, Phys. Rev. C 76, 064316 (2007).

[41] G. C. Strinati, P. Pieri, G. Röpke, P. Schuck, and M. Urban, Phys. Rep. 738, 1 (2018).

[42] L. Pisani, A. Perali, P. Pieri, and G. Calvanese Strinati, Phys. Rev. B 97, 014528 (2018).

[43] N. Chamel, S. Goriely, and J.M. Pearson, Phys. Rev. C 80, 065804 (2009).

[44] M. Jin, M. Urban, and P. Schuck, Phys. Rev. C 82, 024911 (2010).

[45] R. Zimmermann and H. Stolz, Phys. Status Solidi B 131, 151 (1985). 